\title{
Effects of nonlinear wave growth on extreme radiation belt electron fluxes
}

\section{$\operatorname{AUTHOR(S):~}$}

Summers, Danny; Tang, Rongxin; Omura, Yoshiharu

\section{CITATION:}

Summers, Danny ...[et al]. Effects of nonlinear wave growth on extreme radiation belt electron fluxes. JOURNAL OF GEOPHYSICAL RESEARCH-SPACE PHYSICS 2011, 116(A10): A10226.

ISSUE DATE:

2011-10

URL:

http://hdl.handle.net/2433/163427

RIGHT:

(C)2011. American Geophysical Union. 


\title{
Effects of nonlinear wave growth on extreme radiation belt electron fluxes
}

\author{
Danny Summers, ${ }^{1,2}$ Rongxin Tang, ${ }^{1}$ and Yoshiharu Omura ${ }^{3}$ \\ Received 25 February 2011; revised 7 July 2011; accepted 3 August 2011; published 29 October 2011.
}

[1] We present new relativistic formulae for the self-limiting electron integral and differential fluxes in a planetary radiation belt. The formulae depend on the relativistic particle anisotropy, the power gain of electromagnetic whistler mode waves assumed to be generated near the equator, and the associated length scale for convective wave growth. The theoretical limit for the electron differential flux is compared with measured fluxes at geosynchronous orbit. Next we incorporate the effects of nonlinear wave growth into the calculation of extreme radiation belt electron fluxes. We assume that whistler mode waves undergo a linear growth phase, during which the frequency is constant, followed by a nonlinear growth phase during which the frequency is time increasing. We determine the total power gain $G_{T O T}$ as the sum of the linear wave gain $g_{L}$ during linear growth and the nonlinear wave gain $g_{N}$ during nonlinear growth. $G_{T O T}$ depends on the maximum values of the linear and nonlinear wave growth rates at the equator and is hence a function of the energetic electron number density $N_{h}$. For a specified value of $G_{T O T}$, we can determine the value of $N_{h}$ that corresponds to an extreme value for the electron (integral/ differential) flux. Our new result for the extreme electron flux in the nonlinear regime can be regarded as a generalization of the Kennel-Petschek limit, which is based on linear wave growth. The extreme value for the electron flux depends on the parameters that characterize the plasma and the assumed particle distribution, as well as the convective length scales $\lambda_{L} H$ and $\lambda_{N} H$ for linear and nonlinear wave growth.

Citation: Summers, D., R. Tang, and Y. Omura (2011), Effects of nonlinear wave growth on extreme radiation belt electron fluxes, J. Geophys. Res., 116, A10226, doi:10.1029/2011JA016602.

\section{Introduction}

[2] It is well known that relativistic $(>1 \mathrm{MeV})$ electrons can be generated in Earth's outer radiation zone $(3<L<7)$ during geomagnetically disturbed periods [e.g., Baker et al., 1997, 1998; Reeves et al., 1998] and that these "killer" electrons are potentially hazardous to orbiting spacecraft [e.g., Fennell et al., 2000; Baker, 2002]. There is much motivation, therefore, to understand the physical processes that control radiation belt particle dynamics. During the past decade or so, there has been considerable research effort to identify and quantify the acceleration, loss and transport mechanisms of radiation belt particles [e.g., see Friedel et al., 2002; Horne, 2002; O'Brien et al., 2003; Thorne et al., 2005a; Summers et al., 2007a, 2007b; Millan and Thorne, 2007; Shprits et al., 2008; Hudson et al., 2008; Baker and Kanekal, 2008, and references therein].

\footnotetext{
${ }^{1}$ Department of Mathematics and Statistics, Memorial University of Newfoundland, St. John's, Newfoundland, Canada.

${ }^{2}$ School of Space Research, Kyung Hee University, Yongin, South Korea.

${ }^{3}$ Research Institute for Sustainable Humanosphere, Kyoto University, Kyoto, Japan.

Copyright 2011 by the American Geophysical Union. 0148-0227/11/2011JA016602
}

[3] Hazards to spacecraft posed by relativistic electrons are often characterized by "worst-case" observed fluxes, i.e., "extreme" or "maximum" fluxes [e.g., Fennell et al., 2000]. Observations of extreme values of energetic electron fluxes at geosynchronous orbit have been reported, for instance, by O'Brien et al. [2007] and Kataoka and Miyoshi [2008]. There appears therefore to be firm experimental evidence for the existence of an upper limit to radiation belt electron fluxes. Candidate mechanisms that may explain the flux limits include the limit to sustained solar wind velocity [O'Brien et al., 2007], which is a key driver of outer zone electron fluxes, and mechanisms internal to the magnetosphere such as the self-limiting flux concept of Kennel and Petschek [1966]. Limiting particle spectra may also be obtained as the steady state solution of the (three-dimensional) kinetic equation for the particle distribution, in which radial transport, acceleration and loss processes are included. In a more restricted study, Summers and Ma [2000] obtained limiting spectra for outer zone energetic electrons by obtaining a steady state solution of a one-dimensional kinetic equation for the particle energy distribution, with radial transport absent, under geomagnetic storm conditions. The model analyzed by Summers and Ma [2000] was based on the proposed mechanism of Summers et al. [1998] for generating "killer" electrons, namely, electron acceleration due to gyroresonant interaction with whistler mode chorus outside 
the plasmasphere, and electron precipitation loss due to resonant scattering by electromagnetic ion cyclotron waves inside the plasmasphere.

[4] In this paper we address the concept of extreme radiation belt electron flux in two ways:

[5] 1. We further examine the self-limiting flux concept of Kennel and Petschek [1966] in the relativistic regime. In our interpretation of this concept, the limit on stably trapped particle flux is attained in the steady state condition of marginal stability in which whistler mode waves generated near the equator acquire a specified gain over a given convective growth length. In contrast to the nonrelativistic Kennel and Petschek [1966] formulation, Summers et al. [2009] obtained fully relativistic formulae for the limiting electron integral and differential fluxes for a general planetary radiation belt at a given $L$ shell. The theoretical limits on the trapped flux were compared with observed energetic electron fluxes at Earth, Jupiter, and Uranus. In their study on the Kennel-Petschek limit, Mauk and Fox [2010] extended the work of Summers et al. [2009] by assuming an empirically motivated analytical form for the electron differential flux. This form allows more flexible fitting to measured spectral shapes than the power law distribution assumed by Summers et al. [2009]. In the present study, we rework the analysis of Summers et al. [2009] and obtain new explicit formulae for the limiting electron integral and differential fluxes. We then compare the limiting solutions with observed energetic electron fluxes at geosynchronous orbit. Our reanalysis of the Kennel-Petschek limit is presented in section 2.

[6] 2. As an extension of Kennel-Petschek theory which is based on linear wave growth, we present in sections 3-6 a determination of extreme electron flux that includes the effects of nonlinear wave growth. Our analysis here utilizes the nonlinear growth theory of magnetospheric whistler mode emissions developed by Omura et al. [2008, 2009]. We assume that the process of wave growth comprises a linear phase followed by a nonlinear phase. We then calculate the total power gain as the sum of the linear wave gain and the nonlinear wave gain. This enables the calculation of an extreme value for the electron flux, for a specified value of the total wave gain.

[7] We conclude our paper with a brief summary in section 7 .

\section{Self-Limiting Stably Trapped Particle Flux}

[8] As used by Summers et al. [2009], we choose the (equatorial) electron distribution function,

$$
f\left(p_{\|}, p_{\perp}\right)=\frac{J_{\perp}\left(p_{*}\right)}{p^{2}}\left(\frac{p_{*}}{p}\right)^{2 l}(\sin \alpha)^{2 s}, \quad p \geq p_{*}
$$

with $p^{2}=p_{\|}^{2}+p_{\perp}^{2}$, where $p_{\|}=\gamma m_{e} v_{\|}$and $p_{\perp}=\gamma m_{e} v_{\perp}$ are the components of relativistic momentum $\boldsymbol{p}=\gamma m_{e} v, m_{e}$ is the electron rest mass, $\boldsymbol{v}$ is the electron velocity with components $v_{\|}$and $v_{\perp}$, parallel and perpendicular, respectively, to the ambient magnetic field, and $\gamma=\left(1-v^{2} / c^{2}\right)^{-1 / 2}=\left(1+p^{2} /\right.$ $\left.\left(m_{e} c\right)^{2}\right)^{1 / 2}$, with $v^{2}=v_{\|}^{2}+v_{\perp}^{2}$, and $c$ is the speed of light; $\alpha=$ $\tan ^{-1}\left(p_{\perp} / p_{\|}\right)$is the electron pitch angle; $l(>1)$ is the spectral index and $s(>0)$ is the pitch angle index; $p_{*}$ is a minimum value of the momentum to be specified below. The electron differential number flux $J$ is given by

$$
J=p^{2} f .
$$

Hence, $J_{\perp}\left(p_{*}\right)$ in (1) is the perpendicular $(\alpha=\pi / 2)$ differential number flux at $p=p_{*}$. The electron kinetic energy $E$, given by $E /\left(m_{e} c^{2}\right)=\gamma-1$, satisfies the relation

$$
\left(\frac{p}{m_{e} c}\right)^{2}=\left(\frac{E}{m_{e} c^{2}}\right)\left[\left(\frac{E}{m_{e} c^{2}}\right)+2\right] .
$$

By using (1) and (2), the electron integral omnidirectional flux,

$$
I_{4 \pi}\left(E>E_{*}\right)=4 \pi \int_{0}^{1} d(\cos \alpha) \int_{E_{*}}^{\infty} J d E
$$

is given by

$I_{4 \pi}\left(E>E_{*}\right)=2 \pi^{3 / 2} \frac{\Gamma(s+1)}{\Gamma\left(s+\frac{3}{2}\right)} J_{\perp}\left(p_{*}\right)\left(\frac{p_{*}}{m_{e} c}\right)^{2 l}\left(m_{e} c^{2}\right) \int_{\frac{E_{*}}{m_{e} c^{2}}}^{\infty} \frac{d z}{\left(z^{2}+2 z\right)}$,

where

$$
\left(\frac{p_{*}}{m_{e} c}\right)^{2}=\left(\frac{E_{*}}{m_{e} c^{2}}\right)\left[\left(\frac{E_{*}}{m_{e} c^{2}}\right)+2\right],
$$

and $\Gamma$ is the gamma function.

\subsection{Linear Wave Growth Rate}

[9] The cold plasma dispersion relation for electromagnetic $\mathrm{R}$ mode waves propagating parallel to a uniform magnetic field can be written

$$
y^{2}=x^{2}+\frac{x}{a(1-x)}
$$

where

$$
x=\omega /\left|\Omega_{e}\right|, \quad y=c k /\left|\Omega_{e}\right|,
$$

and $a$ is the cold plasma parameter defined by

$$
a=\left|\Omega_{e}\right|^{2} / \omega_{p e}^{2}
$$

where $\omega$ is the (real) wave frequency, $k$ is the (real) wave number, $\left|\Omega_{e}\right|=e B_{0} /\left(m_{e} c\right)$ is the electron gyrofrequency, $\omega_{p e}=\left(4 \pi N_{0} e^{2} / m_{e}\right)^{1 / 2}$ is the plasma frequency, $-e$ is the electron charge, $N_{0}$ is the cold electron number density, and $B_{0}$ is the magnitude of the zeroth-order magnetic field. Then, using a modification of results by Xiao et al. [1998] presented by Summers et al. [2009, Appendix A], we write the temporal growth rate $\omega_{i}$ for parallel-propagating $\mathrm{R}$ mode waves corresponding to the anisotropic electron distribution (1) in the form,

$$
\frac{\omega_{i}}{\left|\Omega_{e}\right|}=\frac{\pi}{2 a y} \cdot \frac{v_{g}}{c} \bar{\eta}_{r e l}\left(\tilde{A}_{r e l}-A_{C}\right)
$$

where

$$
\bar{\eta}_{r e l}=\frac{\pi}{N_{0}} \frac{(1-x)}{y}\left(\frac{p_{*}}{m_{e} c}\right)^{2 l}\left(m_{e} c\right) J_{\perp}\left(p_{*}\right) I_{1}(x, y)
$$


is the fraction of the relativistic particle distribution near resonance,

$$
\tilde{A}_{\text {rel }}=\frac{y}{(1-x)} \frac{I_{2}(x, y)}{I_{1}(x, y)}
$$

is the relativistic pitch angle anisotropy of the resonant particles,

$$
A_{C}=\frac{x}{1-x}
$$

is the critical (minimum) anisotropy required for wave growth $\left(\omega_{i}>0\right)$, and

$$
\frac{v_{g}}{c}=\frac{2 a y(1-x)^{2}}{1+2 a x(1-x)^{2}}
$$

is the normalized group speed of the waves.

[10] $I_{1}(x, y)$ and $I_{2}(x, y)$ are integrals whose forms are given in Appendix A of the present paper. These integrals must in general be evaluated numerically.

\subsection{Limiting Flux}

[11] We assume that whistler mode waves of sufficient power generated at the magnetic equator maintain the stably trapped electron flux close to its limiting value. The wave gain $G$ is given by

$$
G=\int \frac{\omega_{i} d s}{v_{g}},
$$

where the path integral (15) is taken along a flux tube with element $d s$. Condition (15) may be expressed in the approximate form,

$$
G=\frac{\omega_{i} H}{v_{g}},
$$

where $H$ is a specified convective growth length, and in (16) the wave growth rate $\omega_{i}$ and group speed $v_{g}$ are evaluated at the frequency $\omega=\omega_{m}$ at which $\omega_{i}$ maximizes. Substituting (10) and (11) for the wave growth rate $\omega_{i}$ into (16) yields the limiting value of $J_{\perp}\left(p_{*}\right)$ which can be written,

$$
J_{\perp}\left(p_{*}\right)=\frac{B_{0}}{2 \pi^{3} e} \frac{1}{\left(m_{e} c\right)}\left(\frac{m_{e} c}{p_{*}}\right)^{2 l} \frac{y_{m}^{2}}{\left(1-x_{m}\right)} \frac{1}{I_{1}} \frac{G}{\left(\tilde{A}_{r e l}-A_{C}\right) H}
$$

where

$$
x_{m}=\omega_{m} /\left|\Omega_{e}\right|, \quad y_{m}=c k_{m} /\left|\Omega_{e}\right|, \quad k_{m}=k\left(\omega_{m}\right) .
$$

Hence, from (5) and (17), we obtain the limiting omnidirectional electron integral flux,

$$
I_{4 \pi}\left(E>E_{*}\right)=\Lambda \frac{c B_{0}}{\pi^{3 / 2} e} \frac{G}{\left(\tilde{A}_{r e l}-A_{C}\right) H}
$$

where

$$
\Lambda=\frac{\Gamma(s+1)}{\Gamma\left(s+\frac{3}{2}\right)} \frac{y_{m}^{2}}{\left(1-x_{m}\right)} \frac{1}{I_{1}} \int_{\frac{E_{*}}{m_{e^{2}}}}^{\infty} \frac{d z}{\left(z^{2}+2 z\right)^{l}}
$$

and in (17), (19), and (20) the quantities $I_{1}, \tilde{A}_{r e l}, A_{C}$ are evaluated at $x=x_{m}, y=y_{m}$. We identify $p_{*}$ (or $E_{*}$ ) as the minimum electron momentum (or energy) for which gyroresonance can occur with a wave having a positive growth rate. From (10) and (7) we find $\omega_{i}>0$ for $0<\omega<\omega_{*}$, where $x_{*}=\omega_{*} / I \Omega_{e} I$ and $y_{*}=c k_{*} /\left|\Omega_{e}\right|$ satisfy

$$
\tilde{A}_{r e l}\left(x_{*}, y_{*}\right)=A_{C}\left(x_{*}, y_{*}\right)
$$

and

$$
y_{*}^{2}=x_{*}^{2}+\frac{x_{*}}{a\left(1-x_{*}\right)} .
$$

Numerical values for $x_{*}$ and $y_{*}$ are determined by solving equations (21) and (22) simultaneously. We set $\omega=\omega_{*}$, $k=k_{*}, v_{\|}=v_{*}$ and $v_{\perp}=0$ in the relativistic gyroresonance condition,

$$
\omega-k v_{\|}=\left|\Omega_{e}\right| / \gamma,
$$

in order to obtain

$$
\frac{p_{*}}{m_{e} c}=\frac{\gamma_{*} v_{*}}{c}, \frac{E_{*}}{m_{e} c^{2}}=\gamma_{*}-1,
$$

where

$$
\gamma_{*}=\left(1-\frac{v_{*}^{2}}{c^{2}}\right)^{-1 / 2},
$$

and

$$
\frac{v_{*}}{c}=\frac{x_{*} y_{*}-\left(1+y_{*}^{2}-x_{*}^{2}\right)^{1 / 2}}{1+y_{*}^{2}} .
$$

Result (19), with $E_{*}$ given by (24)-(26), is a new explicit form for the limiting value of the omnidirectional electron integral flux for $E>E_{*}$ in the fully relativistic regime. Nonrelativistic approximations corresponding to (17), (19), and (20) are given in Appendix B. Equations (19) and (20) can be shown to be equivalent to equations (26) and (27) of Summers et al. [2009], when $G=3 / 2$. As in previous treatments of the self-limiting flux problem, the derivation of (19) is based on the assumption that wave growth rates are linear. The parameters $x_{*}, x_{m}$, and $E_{*}$ are plotted by Summers et al. [2009] as functions of the parameter $a=$ $\left|\Omega_{e}\right|^{2} / \omega_{p e}^{2}$, for the pitch angle indices $s=0.1,0.5,2.0$, and the fixed spectral index $l=2$; values of $x_{*}, x_{m}$, and $E_{*}$ are also given in tabular form for a specified array of $(s, l)$ values, for the case $a=0.05$.

[12] From (1) and (2) we find the perpendicular differential electron flux is given by

$$
J_{\perp}(p)=J_{\perp}\left(p_{*}\right)\left(\frac{p_{*}}{p}\right)^{2 l}, p \geq p_{*} .
$$

The limiting value of the perpendicular differential electron flux is therefore given by (27) with $J_{\perp}\left(p_{*}\right)$ given by expression (17).

\subsection{Application to Earth}

[13] In the case of Earth, we assume a dipole magnetic field, $B_{0}(L)=B_{E} / L^{3}$ and $H_{P}=L R_{E} / 2$ (where $L$ is magnetic 


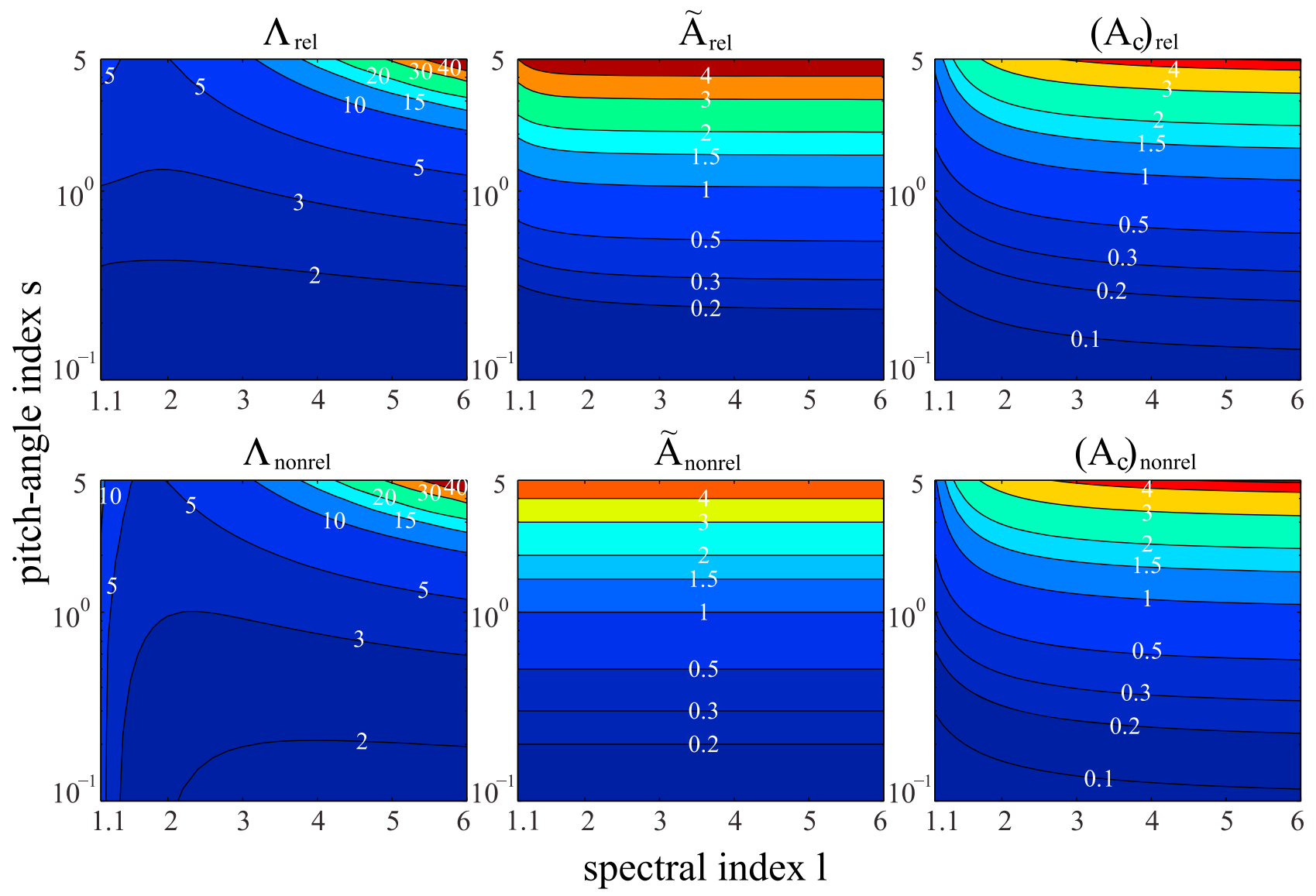

Figure 1. Two-dimensional plots of the parameters $\Lambda(l, s, a), \tilde{A}(l, s, a)$, and $A_{C}(l, s, a)$, in the relativistic and nonrelativistic cases, with $a=\left|\Omega_{e}\right|^{2} / \omega_{p e}^{2}=0.05$.

shell), with $B_{E}=0.312$ gauss and $R_{E}=6.4 \times 10^{8} \mathrm{~cm}$. Then from (19) the limiting omnidirectional electron integral flux is

$$
I_{4 \pi}\left(E>E_{*}\right)=\frac{1.1 \times 10^{10}}{L^{4}} \cdot \frac{\Lambda G}{\left(\tilde{A}_{\text {rel }}-A_{C}\right)} \mathrm{cm}^{-2} \mathrm{~s}^{-1} .
$$

[14] In Figure 1 we show both relativistic and nonrelativistic two-dimensional plots of the parameters $\Lambda(l, s, a)$, $\tilde{A}(l, s, a)$ and $A_{C}(l, s, a)$ for the case $a=0.05$. In particular, we see that $\Lambda$ can be weakly or strongly dependent on the values chosen for $l$ and $s$ subject to the region of $(l, s)$ parameter space considered. For a given $l$ value, $\Lambda, \tilde{A}, \tilde{A}$ and $A_{C}$ are increasing functions of $s$. For a given $s$ value, $\tilde{A}$ and $A_{C}$ are weakly dependent on $l$ if $l>2$. Complementary to Figure 1, we show in Figure 2 plots of $\Lambda, A$, and $A_{C}$ as functions of $s$ for the cases $l=1.1,2.0,4.0$, with $a=0.05$. The relativistic and nonrelativistic values of $\tilde{A}$ and $A_{C}$ are in approximate agreement over most of $(l, s)$ parameter space. Significant differences between $\Lambda_{r e l}$ and $\Lambda_{\text {nonrel }}$ occur for some values of $l$ and $s$, e.g., $1.1<l<4,0.1<s<0.5$.

[15] In Figure 3 we plot the relativistic and nonrelativistic forms of $\Lambda, \tilde{A}$, and $A_{C}$ as functions of $a$ for $s=0.1,0.5,2.0$, with $l=2$. In all cases considered, the nonrelativistic forms of $\Lambda, \tilde{A}$, and $A_{C}$ are weakly dependent on $a$. For $a<0.05$ the relativistic forms of $\tilde{A}$ and $A_{C}$ are weakly dependent on $a$ and reasonably well approximated by the corresponding nonrelativistic forms. For $s>1$ and $a<0.05$, the relativistic form of $\Lambda$ is likewise weakly dependent on $a$ and reasonably approximated by its nonrelativistic form. For $a>0.05, \Lambda, \tilde{A}$, and $A_{C}$ are all decreasing functions of $a$.

[16] In Figure 4 we plot $\Lambda, \tilde{A}$, and $A_{C}$ as functions of $L$, for $s=0.1,0.5,2.0$, with $l=2$. We have assumed the electron number density $N_{0}=124(3 / L)^{4} \mathrm{~cm}^{-3}$ [Sheeley et al., 2001] and a dipole magnetic field in the Earth's trough region. The nonrelativistic forms of $\Lambda, \tilde{A}$, and $A_{C}$ are weakly dependent on $L$. The relativistic forms of $\Lambda, \tilde{A}$, and $A_{C}$ are weakly increasing functions of $L ; \tilde{A}$ and $A_{C}$ are reasonably approximated by their nonrelativistic forms.

[17] We compare our limiting solution (27) with the measured electron differential flux at geosynchronous orbit ( $L=6.6$ ) published by O'Brien et al. [2007]. We choose $G=6, N_{0}=5.29 \mathrm{~cm}^{-3}, a=0.0216, l=1.9$ and we consider $s=0.1,0.2,0.3,0.5$. The results of our comparison are shown in Figure 5. We have set $l=1.9$ so as to best match the slope of our solution with slopes of the data curves. Since experimental values of $s$ associated with the flux measurements of $O^{\prime}$ Brien et al. [2007] were not available, we choose the above $s$ values so as to match the solutions with the data profiles for the three different averaging times. Typical measured $s$ values in Earth's outer radiation belt cover the range $0<s<1.5$ [Thorne et al., 2005b]. From Figure 5 it is clear that some of the data profiles agree remarkably well with the limiting solutions, dependent on 

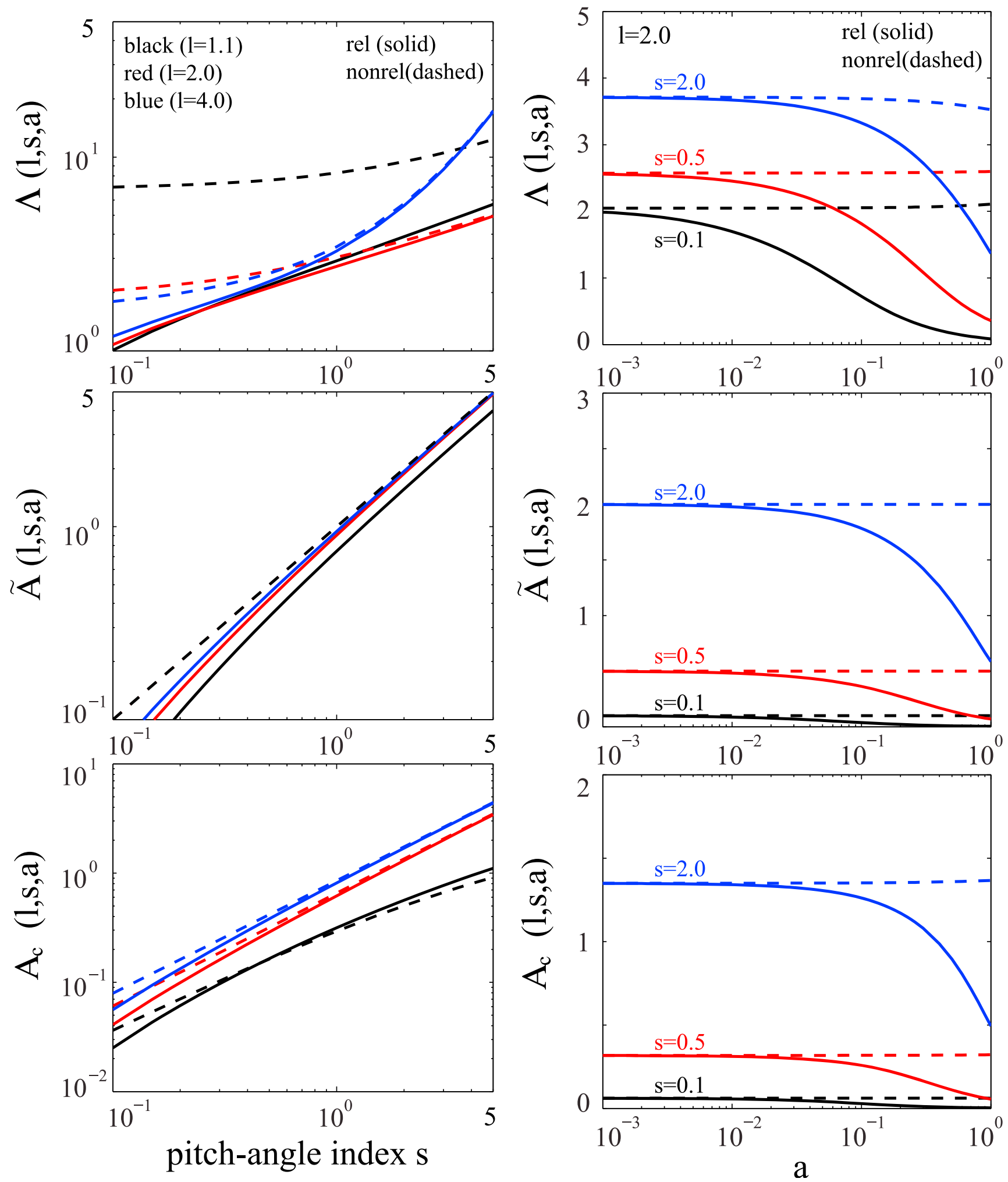

Figure 2. Relativistic and nonrelativistic values of the parameters $\Lambda(l, s, a), \tilde{A}(l, s, a)$, and $A_{C}(l, s, a)$ as functions of the pitch angle index $s$, for $a=\left|\Omega_{e}\right|^{2} / \omega_{p e}^{2}=0.05$ and the indicated values of $l$.

Figure 3. Relativistic and nonrelativistic values of the parameters $\Lambda(l, s, a), \tilde{A}(l, s, a)$, and $A_{C}(l, s, a)$ as functions of the parameter $a=\left|\Omega_{e}\right|^{2} / \omega_{p e}^{2}$, for $l=2$, and the indicated values of $s$. 

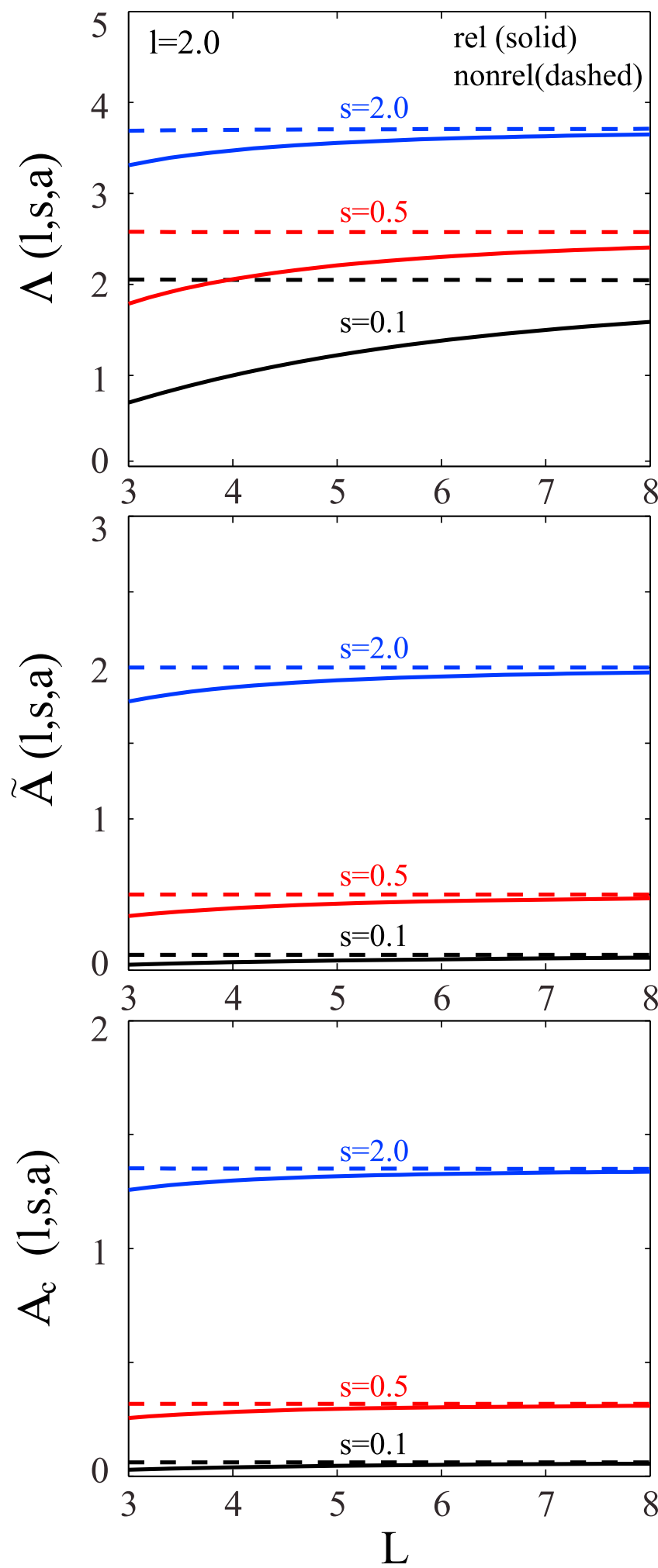

Figure 4. Relativistic and nonrelativistic values of the parameters $\Lambda(l, s, a), \tilde{A}(l, s, a)$, and $A_{C}(l, s, a)$ as functions of $L$ shell, for $l=2$, and the indicated values of $s$. The electron density model $N_{0}=124(3 / L)^{4} \mathrm{~cm}^{-3}$ [Sheeley et al., 2001] and a dipole magnetic field in the Earth's trough region are assumed. the averaging time and chosen $s$ value. A more general or definitive conclusion from this comparison of data with limiting solutions cannot be made since experimental $s$ values were not known.

\section{Nonlinear Wave Growth Effects}

[18] We first consider the distribution $F$ of hot energetic electrons given by

$$
\begin{aligned}
F\left(p_{\|}, p_{\perp}\right)= & \frac{N_{h}}{2 \pi p_{c}} \frac{(2 l-1) \Gamma(l+s+1)}{\Gamma(s+1) \Gamma(l)} \frac{1}{p^{2}}\left(\frac{p_{c}}{p}\right)^{2 l}(\sin \alpha)^{2 s}, \\
& p \geq p_{c} .
\end{aligned}
$$

Distribution (29) is similar to distribution (1) except that the minimum momentum $p_{c}$ is arbitrary, and $F$ has been normalized using $\int F\left(p_{\|}, p_{\perp}\right) d^{3} p=N_{h}$ where $N_{h}$ is the hot electron number density. By following Summers et al. [2009, Appendix A], the relativistic linear temporal growth rate for field-aligned electromagnetic $\mathrm{R}$ mode waves corresponding to distribution (29) can be written

$$
\begin{aligned}
\frac{\omega_{i}}{\left|\Omega_{e}\right|}= & \frac{\pi}{2} \frac{(2 l-1) \Gamma(l+s+1)}{\Gamma(s+1) \Gamma(l)}\left(\frac{N_{h}}{N_{0}}\right)\left(\frac{p_{c}}{m_{e} c}\right)^{2 l-1} \frac{(1-x)^{2}}{\left[1+2 a x(1-x)^{2}\right]} \\
& \cdot\left\{I_{2}(x, y)-\frac{x}{y} I_{1}(x, y)\right\},
\end{aligned}
$$

where the integrals $I_{1}, I_{2}$ are given by (A1) and (A2). The nonrelativistic approximation of (30) is

$$
\begin{aligned}
\frac{\omega_{i}}{\left|\Omega_{e}\right|}= & \frac{\pi}{2}(2 l-1)\left(\frac{N_{h}}{N_{0}}\right)\left(\frac{p_{c}}{m_{e} c}\right)^{2 l-1} \frac{(1-x)^{2}}{\left[1+2 a x(1-x)^{2}\right]} \\
& \cdot\left(\frac{y}{1-x}\right)^{2 l-1}\left[s-\frac{x}{1-x}\right]
\end{aligned}
$$

[19] In Figure 6, for a range of $N_{h} / N_{0}$ values, we plot the relativistic linear growth rate (30) and nonrelativistic linear growth rate (31) for the case $s=0.3, l=1.5, p_{c} /\left(m_{e} c\right)=0.32$, and we have set $a=0.0625$ which corresponds to $N_{0}=$ $44 \mathrm{~cm}^{-3}$ at $L=3.9$ by using the Sheeley et al. [2001] density model. For a given $N_{h} / N_{0}$ value, the relativistic and nonrelativistic growth rates are markedly different, and the maximum growth rate in the nonrelativistic approximation is approximately 2.1 times the relativistic maximum growth rate.

[20] Omura et al. [2008, 2009] have recently developed a nonlinear wave growth theory for magnetospheric whistler mode chorus emissions. According to this theory, the nonlinear growth rate $\Gamma_{N}$ for field-aligned whistler mode (R mode) waves of frequency $\omega(t)$ and wave magnetic field amplitude $B_{w}(t)$ is given by the relation,

$$
\frac{d B_{w}}{d t}=\Gamma_{N} B_{w},
$$

with 


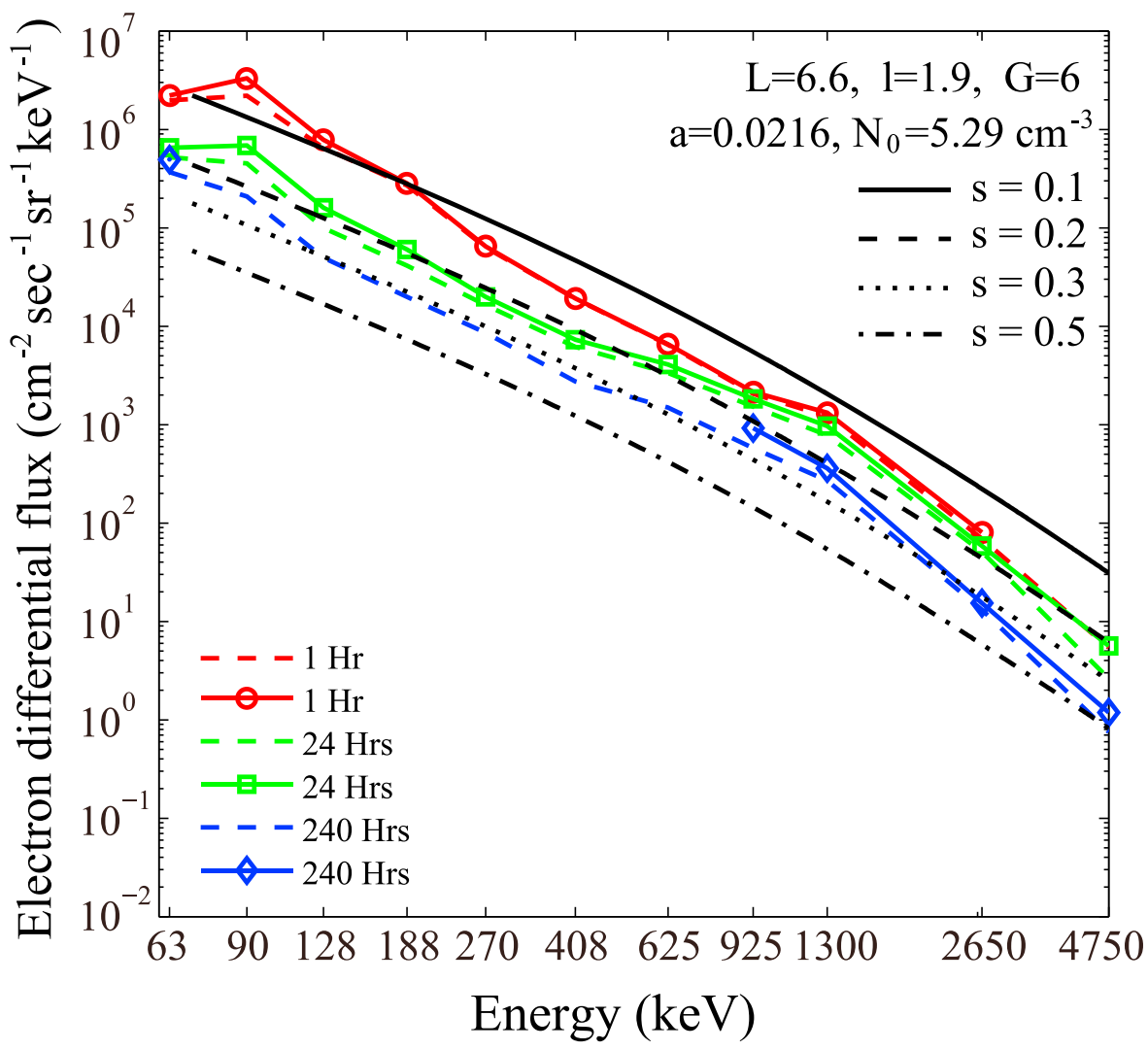

Figure 5. Maxima (for three different averaging times) for the electron differential flux at geosynchronous orbit [O'Brien et al., 2007], together with limiting solutions (in black).

$$
\Gamma_{N}=\frac{4 \sqrt{2} \pi e^{3 / 2}}{m_{e}^{1 / 2} c}\left(\frac{\xi \chi}{B_{w} \omega \tilde{\gamma}_{R}}\right)^{1 / 2} V_{g} V_{\perp 0}^{5 / 2} Q \chi\left(m_{e}^{2} \tilde{G}\right),
$$

and

$$
\begin{gathered}
\xi^{2}=\frac{\omega\left(\left|\Omega_{e}\right|-\omega\right)}{\omega_{p e}^{2}}, \chi^{2}=\frac{1}{1+\xi^{2}}, \\
\frac{V_{g}}{c}=\frac{\xi}{\chi}\left[\xi^{2}+\frac{\Omega_{e}}{2\left(\left|\Omega_{e}\right|-\omega\right)}\right]^{-1}, \\
\tilde{\gamma}_{R}=\left[1-\left(\frac{\tilde{V}_{R}}{c}\right)^{2}-\left(\frac{V_{\perp 0}}{c}\right)^{2}\right]^{-1}, \frac{\tilde{V}_{R}}{c}=\chi \xi\left(1-\frac{\left|\Omega_{e}\right|}{\tilde{\gamma}_{R} \omega}\right),
\end{gathered}
$$

where $V_{g}(t)$ is the wave group speed, $\tilde{\gamma}_{R}(t)$ is the resonant Lorenz factor, $\tilde{V}_{R}(t)$ is the resonant parallel particle velocity, $V_{\perp 0}$ (=constant) is the average perpendicular particle velocity, and $Q$ is the dimensionless factor that represents the depth of the electromagnetic electron hole within which nonlinear particle trapping takes place. The quantity $\tilde{G}$ in (33) is a measure of the average value of the assumed hot electron distribution $F_{t}$ trapped by the wave. The trapped distribution $F_{t}$ is often assumed to be a simple electron ring distribution,

$$
F_{t}\left(p_{\|}, p_{\perp}\right)=\Phi\left(p_{\|}\right) \delta\left(p_{\perp}-p_{\perp 0}\right)
$$

with

$$
p_{\perp 0}=\gamma_{0} m_{e} V_{\perp 0}, \quad \gamma_{0}=\left(1-\frac{V_{\perp 0}^{2}}{c^{2}}\right)^{-1 / 2}
$$

where $\delta$ is the Dirac delta function, and $\Phi$ is a function of parallel particle momentum $p_{\|}$only; $\tilde{G}$ is given by

$$
\tilde{G}=\left[\int F_{t} d p_{\perp}\right]_{p_{\|}=\tilde{p}_{R}}=\Phi\left(\tilde{p}_{R}\right)
$$

where $\tilde{p}_{R}=\tilde{\gamma}_{R} m_{e} \tilde{V}_{R}$.

[21] Here, we approximate distribution (29) by the ring distribution

$$
F_{t}\left(p_{\|}, p_{\perp}\right)=\frac{N_{h}}{\pi} \frac{c_{1}}{p_{c}^{2}}\left(\frac{p_{c}}{p_{\|}}\right)^{2(1+l+s)} \cdot \delta\left(p_{\perp}-p_{\perp 0}\right), \quad p_{\|} \geq p_{c},
$$

where

$$
\begin{gathered}
p_{\perp 0}=c_{2} p_{c}, \\
c_{1}=\frac{(l-1)(2 l+2 s+1) \Gamma(s+1) \Gamma(l)}{2(2 l-1) \Gamma\left(s+\frac{3}{2}\right) \Gamma\left(l-\frac{1}{2}\right)}, \\
c_{2}=\frac{(2 l-1) \Gamma\left(s+\frac{3}{2}\right) \Gamma\left(l-\frac{1}{2}\right)}{2(l-1) \Gamma(s+1) \Gamma(l)} .
\end{gathered}
$$




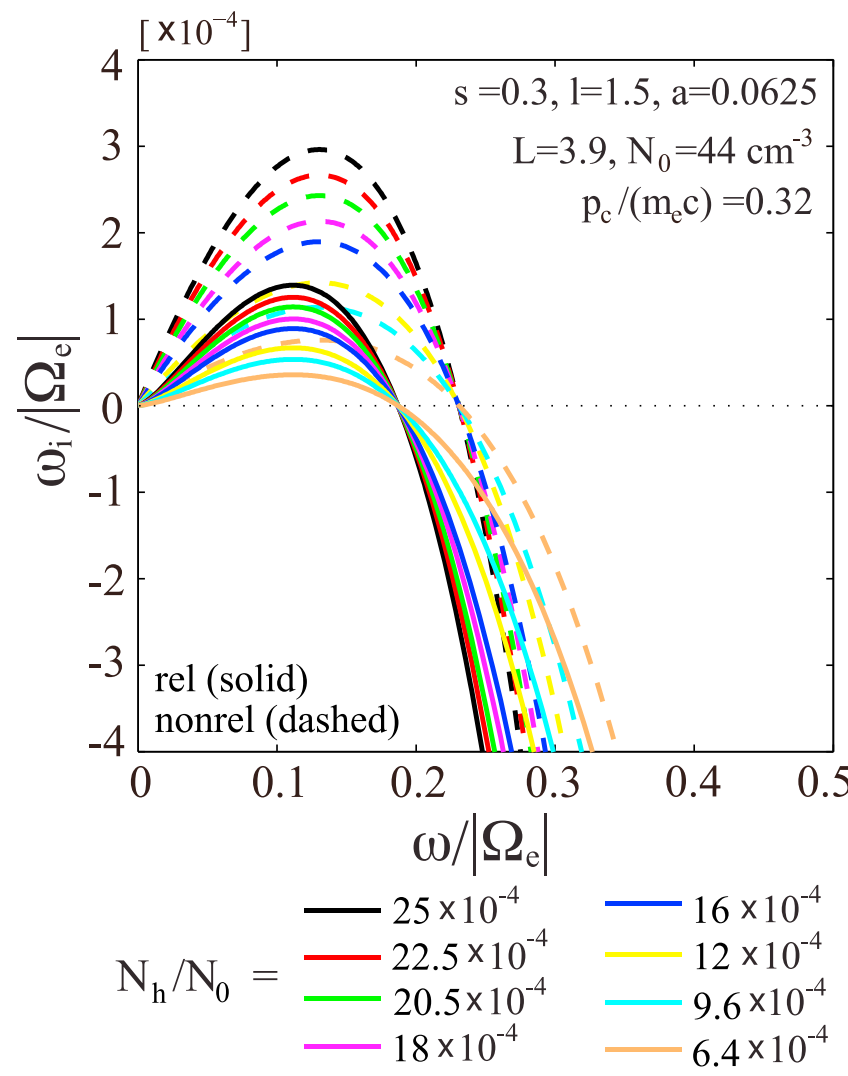

Figure 6. Linear whistler mode wave growth rates (30) and (31) corresponding to the particle distribution (29) for the indicated values of the hot electron number density $N_{h}$.

Hence, from (37), (39), and (40) it follows that

$$
\tilde{G}=\frac{N_{h}}{\pi} \frac{c_{1}}{p_{c}^{2}}\left(\frac{p_{c}}{\tilde{p}_{R}}\right)^{2(1+l+s)} .
$$

[22] The pair of coupled differential equations for $\tilde{B}_{w}=$ $B_{w}(t) / B_{0}$ and $\tilde{\omega}=\omega(t) /\left|\Omega_{e}\right|$, corresponding to the "chorus equations" (40) and (41) given by Omura et al. [2009], are

$$
\begin{gathered}
\frac{\partial \tilde{B}_{w}}{\partial \tilde{t}}=\frac{\Gamma_{N}}{\left|\Omega_{e}\right|} \tilde{B}_{w}-5 \frac{s_{2}}{s_{0}} \frac{V_{g}}{c} \frac{\tilde{a}}{\tilde{\omega}}, \\
\frac{\partial \tilde{\omega}}{\partial \tilde{t}}=\frac{2 s_{0}}{5 s_{1}} \tilde{\omega} \tilde{B}_{w},
\end{gathered}
$$

with $\tilde{t}=\left|\Omega_{e}\right| t$, and

$$
\begin{gathered}
s_{0}=\frac{\chi}{\xi} \frac{V_{\perp 0}}{c}, \\
s_{1}=\tilde{\gamma}_{R}\left(1-\frac{\tilde{V}_{R}}{V_{g}}\right)^{2}, \\
s_{2}=\frac{1}{2 \xi \chi}\left\{\tilde{\gamma}_{R} \tilde{\omega}\left(\frac{V_{\perp 0}}{c}\right)^{2}-\left[2+\chi^{2} \frac{\left(1-\tilde{\gamma}_{R} \tilde{\omega}\right)}{(1-\tilde{\omega})}\right] \frac{\tilde{V}_{R} V_{P}}{c^{2}}\right\},
\end{gathered}
$$

$$
\tilde{a}=\frac{4.5}{\left(L R_{E}\right)^{2}}\left(\frac{c}{\Omega_{e}}\right)^{2}, \frac{V_{P}}{c}=\chi \xi
$$

where $V_{P}(t)$ is the wave phase speed.

[23] The wave equations (45) and (46) are in general valid at the magnetic equator for wave frequencies in the range $0.1 \leq \tilde{\omega} \leq 0.5$, and for wave amplitudes such that $\tilde{B}_{w}(t)>\tilde{B}_{t h}$, where $\widetilde{B}_{t h}=B_{t h} / B_{0}$ is the normalized threshold amplitude given by

$$
\tilde{B}_{t h}=\frac{25 \pi^{2}}{2}\left(\frac{\tilde{a} s_{2}}{Q}\right)^{2} \frac{\tilde{\gamma}_{R} \xi}{\tilde{\omega} \chi^{5}}\left(\frac{c}{V_{\perp 0}}\right)^{3}\left(\frac{N_{0}}{N_{h}}\right)^{2} \frac{a^{2} \gamma_{0}^{4}}{c_{1}^{2} c_{2}^{4}}\left(\frac{\tilde{p}_{R}}{p_{c}}\right)^{4(1+l+s)},
$$

where the parameter $a$ is given by (9). Expression (51) is a useful analytical approximation to the threshold wave amplitude. The exact threshold value (which may be greater than or less than (51)) must be obtained numerically.

[24] Solutions to equations (45) and (46) depend on the parameters $l, s, a, N_{h} / N_{0}, L, Q$, and $p_{c}$ (or $V_{\perp 0}$ ), as well as on the initial values $\tilde{B}_{w}(0), \tilde{\omega}(0)$. The parameters $p_{c}$ and $V_{\perp 0}$ are related by equations (38) and (41) and so cannot be assigned independently.

[25] We solve equations (45) and (46) for the parameter values chosen in Figure 6, namely $s=0.3, l=1.5, a=0.0625$, $p_{c} /\left(m_{e} c\right)=0.32$ (which corresponds to $V_{\perp 0} / c=0.605$ ), and we set $Q=0.5$. As initial conditions, we choose $\tilde{B}_{w}(0)=$ $B_{w}(0) / B_{0}$ to be slightly greater than the numerical threshold value, and we take $\omega(0)$ to be the frequency $\omega_{m}$ at which the relativistic linear growth rate (30) maximizes. In Figure 7 we show the solutions for the wave amplitude $B_{w} / B_{0}$ and the wave frequency $\omega /\left|\Omega_{e}\right|$, together with the corresponding solutions for the nonlinear growth rate $\Gamma_{N} /\left|\Omega_{e}\right|$, for each of the $N_{h} / N_{0}$ values specified in Figure 6. The solutions are characteristic of rising tone whistler mode emissions in which the frequency increases in time [see Omura et al., 2009, section 5]. The nonlinear growth rate increases to a maximum value and then decreases, as time and frequency increase. Comparing Figures 6 and 7, we find that for a given $N_{h} / N_{0}$ value the maximum nonlinear growth rate is about 26-64 times the maximum relativistic linear growth rate. The minimum electron resonant energy $E_{\min }$ at wave frequencies at which the maximum linear and nonlinear wave growth occurs is typically in the range tens to hundreds of $\mathrm{keV}$ for $a=0.0625$; see Figure 8, which was constructed using Summers et al. [2007a, forumula (16)].

\section{Total Wave Gain}

[26] In order to estimate the total power gain during wave growth, we consider the linear growth phase, during which the wave frequency is constant, followed by a nonlinear growth phase with a rising $(\partial \omega / \partial t>0)$ frequency. Many raytracing studies [e.g., Nagano et al., 1998; Chum et al., 2007; Li et al., 2009] have addressed the linear growth phase, and recent particle simulations have analyzed the evolution of wave growth from the linear to nonlinear phases [e.g., Omura and Summers, 2006; Omura et al., 2008; Katoh and Omura, 2011; Omura and Nunn, 2011]. Motivated by these simulation studies, we extend the linear wave gain expression (15) and write the total wave gain in the form, 

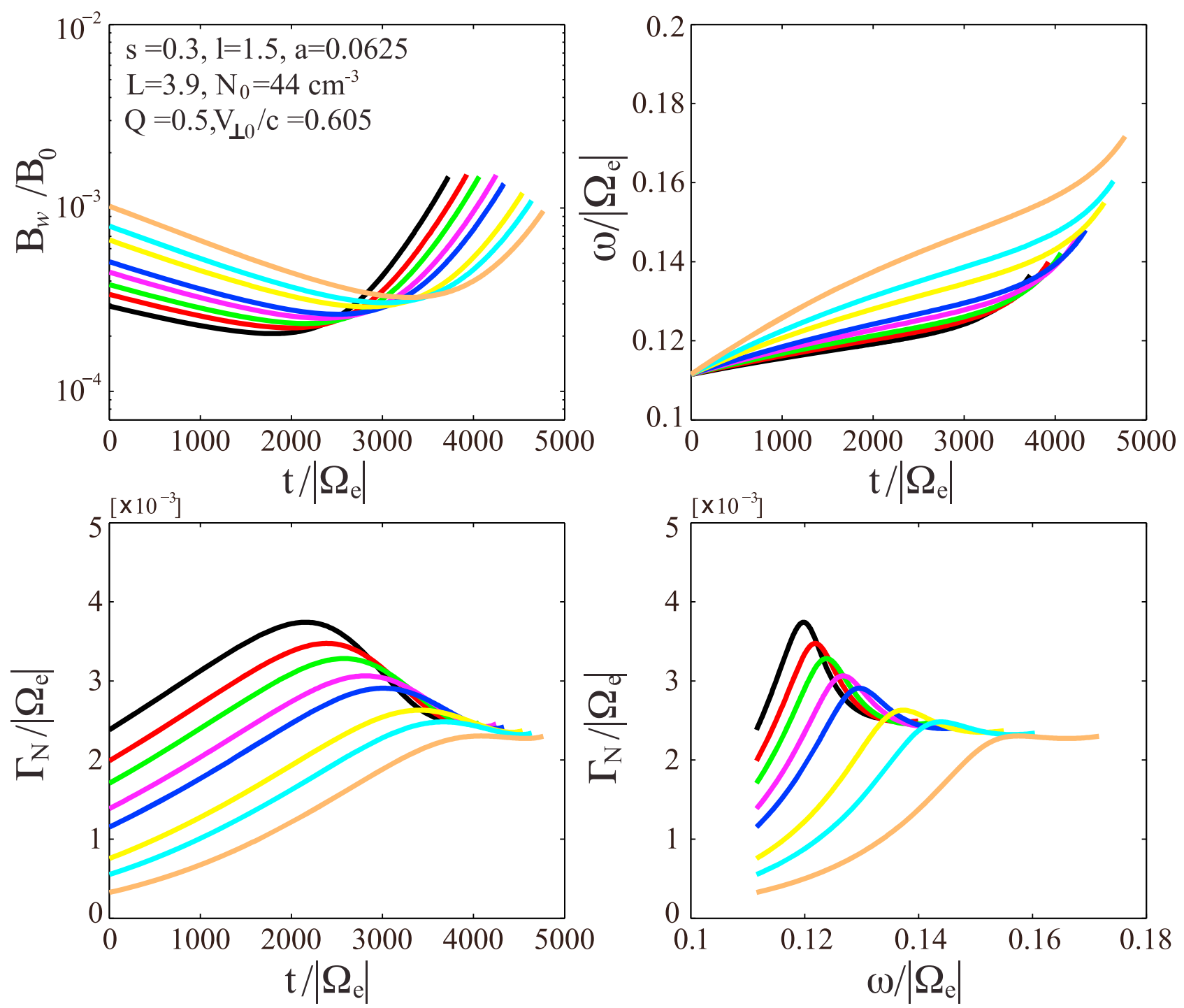

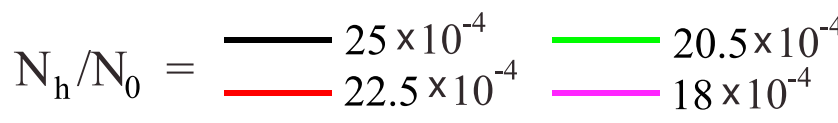

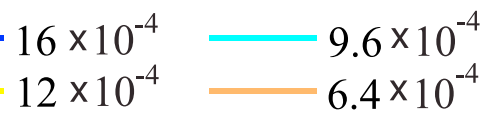

Figure 7. Solutions to the whistler mode wave equations (45) and (46) at the equator, corresponding to the particle distribution (40) for the indicated values of the hot electron number density $N_{h} ; B_{w} / B_{0}$ is the normalized wave amplitude, $\omega /\left|\Omega_{e}\right|$ is the normalized wave frequency, and $\Gamma_{N} /\left|\Omega_{e}\right|$ is the normalized nonlinear wave growth rate.

$$
G_{T O T}=\int_{-H_{1}}^{0}\left[\frac{\omega_{i}}{v_{g}}\right]_{\omega=\omega_{m}} d s+\int_{0}^{H_{2}}\left[\frac{\Gamma_{N}}{v_{g}}\right]_{\omega=\omega_{M}} d s,
$$

where $H_{1}$ is the convective length scale over which linear wave growth takes place, and $\mathrm{H}_{2}$ is the convective length scale over which nonlinear wave growth takes place; integration is carried out along a magnetic field line; $\omega=\omega_{m}$ is the frequency at which the linear temporal growth rate $\omega_{i}$ maximizes at the equator, and $\omega=\omega_{M}$ is the frequency at which the nonlinear temporal growth rate $\Gamma_{N}$ maximizes at the equator. From (52), the maximum wave gain is

$$
G_{T O T}=H_{1}\left[\frac{\omega_{i}}{v_{g}}\right]_{\omega=\omega_{m}}+H_{2}\left[\frac{\Gamma_{N}}{v_{g}}\right]_{\omega=\omega_{M}} .
$$

[27] We set $H_{1}=\lambda_{L} H, H_{2}=\lambda_{N} H$ where $H=L R_{E} / 2$ and $\lambda_{L}, \lambda_{N}$ are scalars, and we hence write

$$
G_{T O T}=g_{L}+g_{N},
$$

with

$$
g_{L}=\lambda_{L} H\left[\frac{\omega_{i}}{v_{g}}\right]_{\omega=\omega_{m}}, \quad g_{N}=\lambda_{N} H\left[\frac{\Gamma_{N}}{v_{g}}\right]_{\omega=\omega_{M}} .
$$




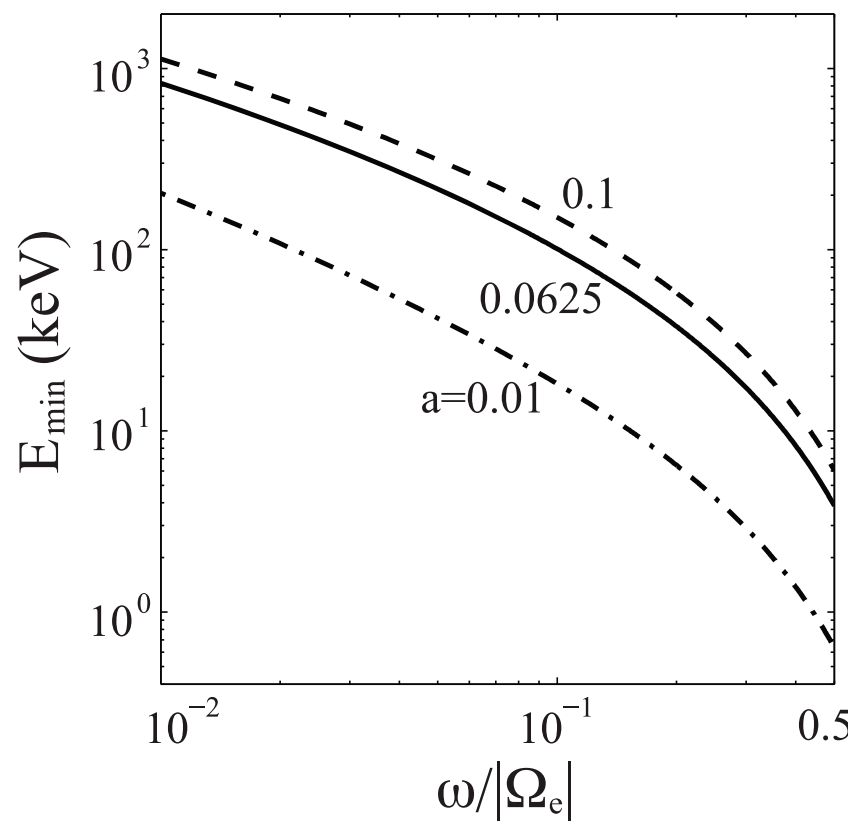

Figure 8. Minimum energy $E_{\min }(\mathrm{keV})$ for electron gyroresonance with R mode waves, for specified values of $a=\left|\Omega_{e}\right|^{2} / \omega_{p e}^{2}$.

[28] From (30) and (55), we find

$$
\begin{aligned}
\frac{g_{L}}{\lambda_{L}}= & \frac{4.63 \times 10^{4}}{L^{2}} \frac{N_{h}}{N_{0}} \frac{1}{a} \frac{(2 l-1) \Gamma(l+s+1)}{\Gamma(s+1) \Gamma(l)}\left(\frac{p_{c}}{m_{e} c}\right)^{2 l-1} \\
& \cdot\left\{\frac{y_{m} I_{2}\left(x_{m}, y_{m}\right)-x_{m} I_{1}\left(x_{m}, y_{m}\right)}{y_{m}^{2}}\right\},
\end{aligned}
$$

and from (33), (44), and (55),

$\frac{g_{N}}{\lambda_{N}}=\frac{2.66 \times 10^{4}}{L^{2}} \frac{N_{h}}{N_{0}} \frac{c_{1} c_{2}^{2}}{a}\left(\frac{\xi}{\tilde{B}_{w} \tilde{\omega} \tilde{\gamma}_{R}}\right)^{1 / 2} \frac{Q \chi^{3 / 2}}{\gamma_{0}^{2}}\left(\frac{V_{\perp 0}}{c}\right)^{1 / 2}\left(\frac{p_{c}}{\tilde{p}_{R}}\right)^{2(1+l+s)}$.

Results (56) and (57) give the maximum linear convective wave gain $g_{L}$ and the maximum nonlinear convective wave gain $g_{N}$, once the scalars $\lambda_{L}, \lambda_{N}$ are known. The relevant quantities on the right-hand side of (57) are evaluated at the frequency $\omega=\omega_{M}$ at which $\Gamma_{N}$ maximizes. Frequency $\omega_{m}$ is independent of $N_{h} / N_{0}$, and the linear gain $g_{L}$ is a linear function of $N_{h} / N_{0}$; frequency $\omega_{M}$ is dependent on $N_{h} / N_{0}$, and the nonlinear gain $g_{N}$ is a nonlinear function of $N_{h} / N_{0}$.

[29] For the parameter values adopted in Figures 6 and 7, we plot $g_{N} / \lambda_{N}$ and $g_{L} / \lambda_{L}$ in Figure 9 as functions of $N_{h} / N_{0}$. For a given value of $N_{h} / N_{0}$, we find that $g_{N} / \lambda_{N} \geq 15\left(g_{L} / \lambda_{L}\right)$, approximately. In section 2 , and in the study by Summers et al. [2009], only a linear wave gain is considered and we set $\lambda_{L}=1$. In this case, when $G_{T O T}=g_{L}=3 / 2$ (as assumed by Summers et al. [2009]), we find from Figure 9 that $N_{h} / N_{0}=$ $9.6 \times 10^{-4}$. This value is consistent with the result $J_{\perp}\left(p_{*}\right)=$ $2.4 \times 10^{6} \mathrm{~cm}^{-2} \mathrm{~s}^{-1} \mathrm{sr}^{-1} \mathrm{keV}^{-1}$ obtained from Summers et al. [2009, formula (54)].

\section{Extreme Electron Flux}

[30] Corresponding to distribution (29), the electron integral omnidirectional flux is

$$
I_{4 \pi}\left(E>E_{r}\right)=\frac{\sqrt{\pi}(2 l-1) \Gamma(l+s+1)}{\Gamma\left(s+\frac{3}{2}\right) \Gamma(l)} N_{h} c\left(\frac{p_{c}}{m_{e} c}\right)^{2 l-1} \int_{\frac{E_{r}}{m_{e} c^{2}}}^{\infty} \frac{d z}{\left(z^{2}+2 z\right)^{l}},
$$

where we specify $E_{r}$ as the minimum electron energy for resonant interaction with electromagnetic $\mathrm{R}$ mode waves of frequency $\omega_{r}$, where $\omega_{M}<\omega_{r}<0.5\left|\Omega_{e}\right|$. For a specified value of the total wave gain $G_{T O T}$, a corresponding "extreme" value of $N_{h} / N_{0}$ can be obtained numerically from equations (54)-(57) for given values of $\lambda_{L}$ and $\lambda_{N}$. This can be achieved by plotting $G_{T O T}=g_{L}+g_{N}$ as a function of $N_{h} / N_{0}$, and then estimating from this plot the value of $N_{h} / N_{0}$ that corresponds to the given value of $G_{T O T}$. The integral flux (58) evaluated for the extreme value of $N_{h} / N_{0}$ corresponds to an extreme value of the equatorial electron flux in the nonlinear regime. This extreme flux can be regarded as a generalization in the nonlinear regime of the KennelPetschek self-limiting flux in the linear regime. Computer simulations [e.g., Omura et al., 2008; Hikishima et al., 2009; Omura and Nunn, 2011] reveal that the spatial scales for linear and nonlinear wave growth are comparable, i.e., $\lambda_{L} \approx$ $\lambda_{N} \approx 0.5$. Consequently, in typical cases in which the nonlinear growth rate well exceeds the linear growth rate (Figures 6 and 7) and the consequent nonlinear gain exceeds the linear gain (Figure 9), the corresponding extreme value for the electron flux is much reduced from that predicted by Kennel-Petschek linear theory.

[31] The perpendicular differential electron flux corresponding to distribution (29) is

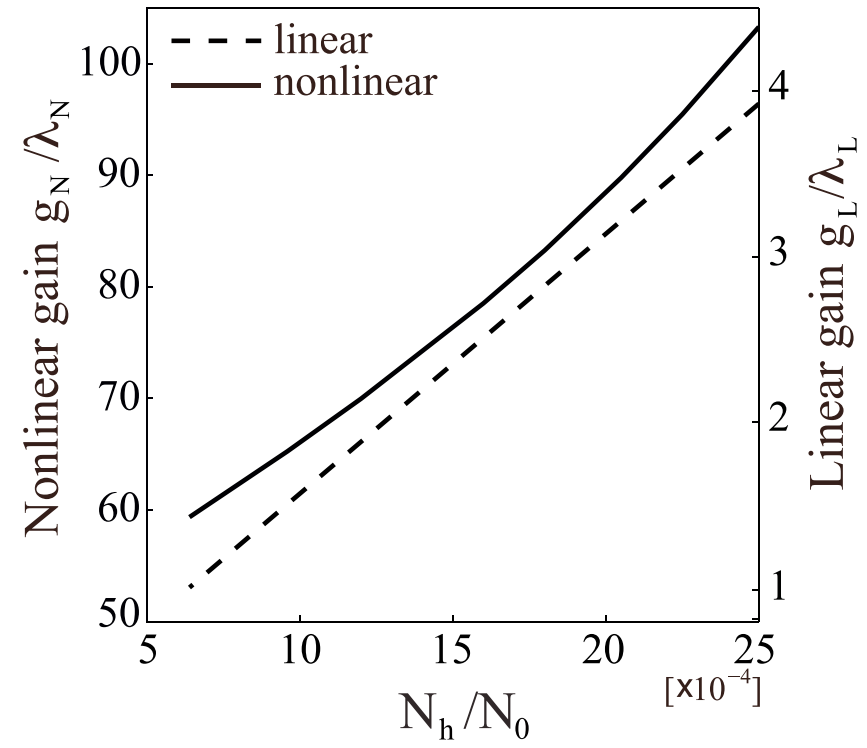

Figure 9. Linear wave gain $g_{L} / \lambda_{L}$ given by (56) and nonlinear wave gain $g_{N} / \lambda_{N}$ given by (57), each plotted as functions of the hot electron number density $N_{h}$, for the parameter values chosen in Figures 6 and 7. 


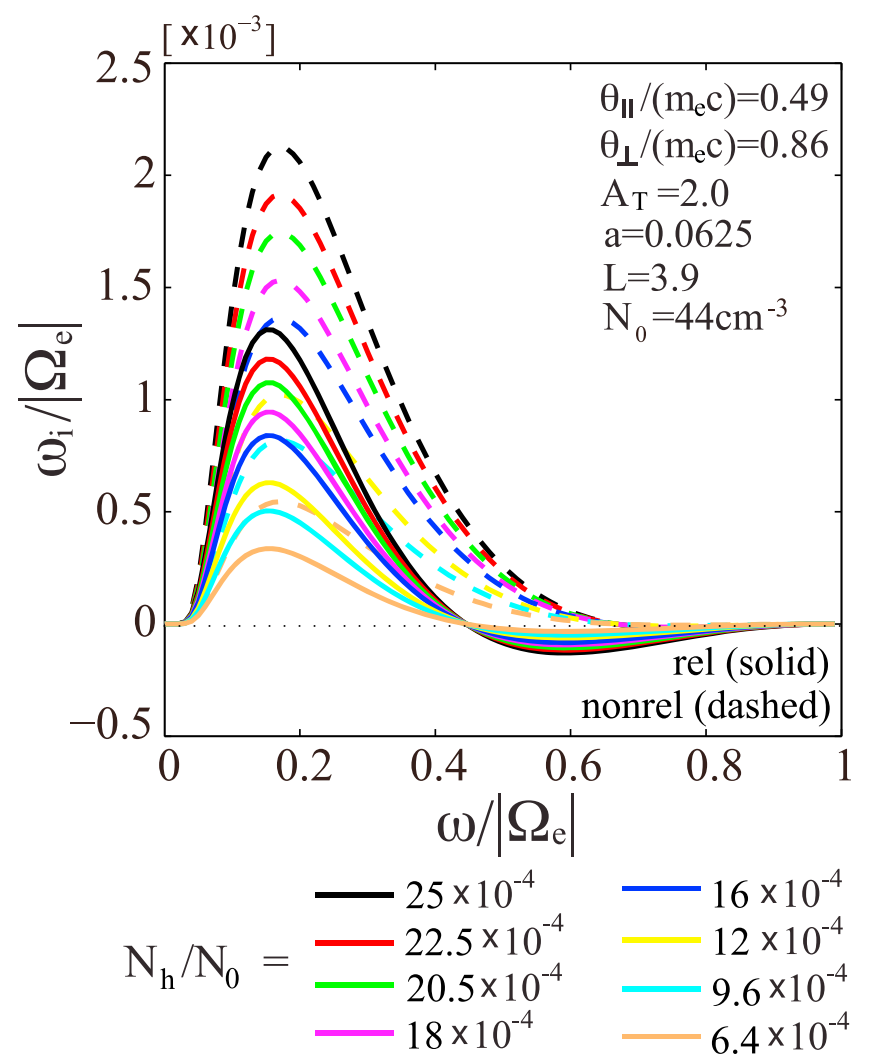

Figure 10. Linear whistler mode wave growth rates (61) and (62) corresponding to the bi-Maxwellian particle distribution (60) for the indicated values of the hot electron number density $N_{h}$.

$$
J_{\perp}(p)=\frac{N_{h}}{2 \pi p_{c}} \frac{(2 l-1) \Gamma(l+s+1)}{\Gamma(s+1) \Gamma(l)}\left(\frac{p_{c}}{p}\right)^{2 l}, \quad p \geq p_{c} .
$$

The extreme value of the perpendicular differential electron flux is therefore given by (59) when $N_{h}$ assumes its extreme value.

\section{Wave Gains for the Bi-Maxwellian and Associated Ring Distribution}

[32] Our analysis of nonlinear wave growth rates and nonlinear wave gain have been based hitherto on the particle distributions (29) and (37). We now consider the bi-Maxwellian distribution,

$$
F^{M}\left(p_{\|}, p_{\perp}\right)=\frac{N_{h}}{\pi^{3 / 2} \theta_{\|} \theta_{\perp}^{2}} \exp \left(-\frac{p_{\|}^{2}}{\theta_{\|}^{2}}-\frac{p_{\perp}^{2}}{\theta_{\perp}^{2}}\right)
$$

which satisfies $\int F^{M}\left(p_{\|}, p_{\perp}\right) d^{3} p=N_{h}$. The relativistic linear temporal growth rate for field-aligned whistler mode waves corresponding to distribution (60) is given by

$$
\frac{\omega_{i}}{\left|\Omega_{e}\right|}=2 \sqrt{\pi} \frac{N_{h}}{N_{0}}\left(\frac{m_{e} c}{\theta_{\|}}\right) \frac{(1-x)^{2}}{\left[1+2 a x(1-x)^{2}\right]}\left\{I_{4}(x, y)-\frac{x}{y} I_{3}(x, y)\right\},
$$

where the integrals $I_{3}, I_{4}$ are given in Appendix $\mathrm{C}$; the nonrelativistic form of (61) is

$$
\begin{aligned}
\frac{\omega_{i}}{\left|\Omega_{e}\right|}= & \frac{\sqrt{\pi}(1-x)^{2}}{\left[1+2 a x(1-x)^{2}\right]} \frac{N_{h}}{N_{0}}\left(\frac{m_{e} c}{\theta_{\|}}\right)\left(\frac{1-x}{y}\right)\left(A_{T}-\frac{x}{1-x}\right) \\
& \cdot \exp \left[-\left(\frac{1-x}{y}\right)^{2}\left(\frac{m_{e} c}{\theta_{\|}}\right)^{2}\right]
\end{aligned}
$$

where $A_{T}=\theta_{\perp}^{2} / \theta_{\|}^{2}-1$ is the thermal anisotropy of the hot energetic electrons.

[33] In Figure 10, for various $N_{h} / N_{0}$ values we plot the relativistic (61) and nonrelativistic (62) linear growth rates for whistler mode waves corresponding to distribution (60) for the parameters $\theta_{\|} /\left(m_{e} c\right)=0.49, \theta_{\perp} /\left(m_{e} c\right)=0.86, A_{T}=$ 2.0 , with $a=0.0625$ which corresponds to $N_{0}=44 \mathrm{~cm}^{-3}$ at $L=3.9$. As in Figure 6, the relativistic and nonrelativistic growth rates differ significantly, and here the maximum nonrelativistic growth rate is 1.6 times the maximum relativistic growth rate.

[34] We approximate (60) by the ring distribution,

$$
F_{t}^{M}\left(p_{\|}, p_{\perp}\right)=\frac{N_{h}}{\pi^{2} \theta_{\|} \theta_{\perp}} \exp \left(-\frac{p_{\|}^{2}}{\theta_{\|}^{2}}\right) \delta\left(p_{\perp}-p_{\perp 0}\right),
$$

where $p_{\perp 0}=\sqrt{\pi} \theta_{\perp} / 2$. The trapped distribution (63) was also used by Omura et al. [2009] in developing their nonlinear wave growth theory. From (37), (39), and (63), we find that the $\tilde{G}$ value corresponding to distribution (63) is

$$
\tilde{G}^{M}=\frac{N_{h}}{\pi^{2} \theta_{\|} \theta_{\perp}} \exp \left(-\frac{\tilde{p}_{R}^{2}}{\theta_{\|}^{2}}\right) .
$$

Hence, the nonlinear growth rate $\Gamma_{N}^{M}$ corresponding to distribution (63) is given by expression (33) with $\tilde{G}$ replaced by $\tilde{G}^{M}$. The wave equations corresponding to distribution (63), namely equations (45) and (46) with $\Gamma_{N}$ replaced by $\Gamma_{N}^{M}$, are in general valid for wave amplitudes such that $\tilde{B}_{w}(t)>\widetilde{B}_{t h}^{M}$, where

$\tilde{B}_{t h}^{M}=50 \pi^{3}\left(\frac{\tilde{a} s_{2}}{Q}\right)^{2} \frac{\tilde{\gamma}_{R}^{3} \xi}{\tilde{\omega} \chi^{5}}\left(\frac{c}{V_{\perp 0}}\right)^{5}\left(\frac{\theta_{\|}}{m_{e} c}\right)^{2}\left(\frac{N_{0}}{N_{h}}\right)^{2} a^{2} \exp \left(\frac{2 \tilde{p}_{R}^{2}}{\theta_{\|}^{2}}\right)$.

In this case, the solutions $\tilde{B}_{w}(t), \tilde{\omega}(t)$ depend on the parameters $a, \theta_{\|}, \theta_{\perp}, N_{h} / N_{0}, L, Q, V_{\perp 0}$ and $\tilde{B}_{w}(0), \tilde{\omega}(0)$. Expression (65) is a useful analytical approximation to the exact threshold wave amplitude which must be obtained numerically.

[35] We solve the wave equations (45) and (46) in the case of distribution (63) and the corresponding nonlinear growth rate $\Gamma_{N}^{M}$, for the parameter values used in Figure 10, with $Q=0.5$ and $V_{\perp 0} / c=0.6$. As initial conditions, we take $B_{w}(0) / B_{0}$ to exceed the numerical threshold value slightly, and we set $\omega(0)$ to be equal to the frequency at which the relativistic linear growth rate (61) maximizes. In Figure 11 we show solutions for $B_{w}, \omega$, and $\Gamma_{N}^{M}$ for the $N_{h} / N_{0}$ values specified in Figure 10. The solutions typify rising tone whistler mode waves and are similar to those in Figure 7. Comparing Figures 10 and 11, we find that the maximum value of the nonlinear growth rate is 4.1-5.6 times the max- 

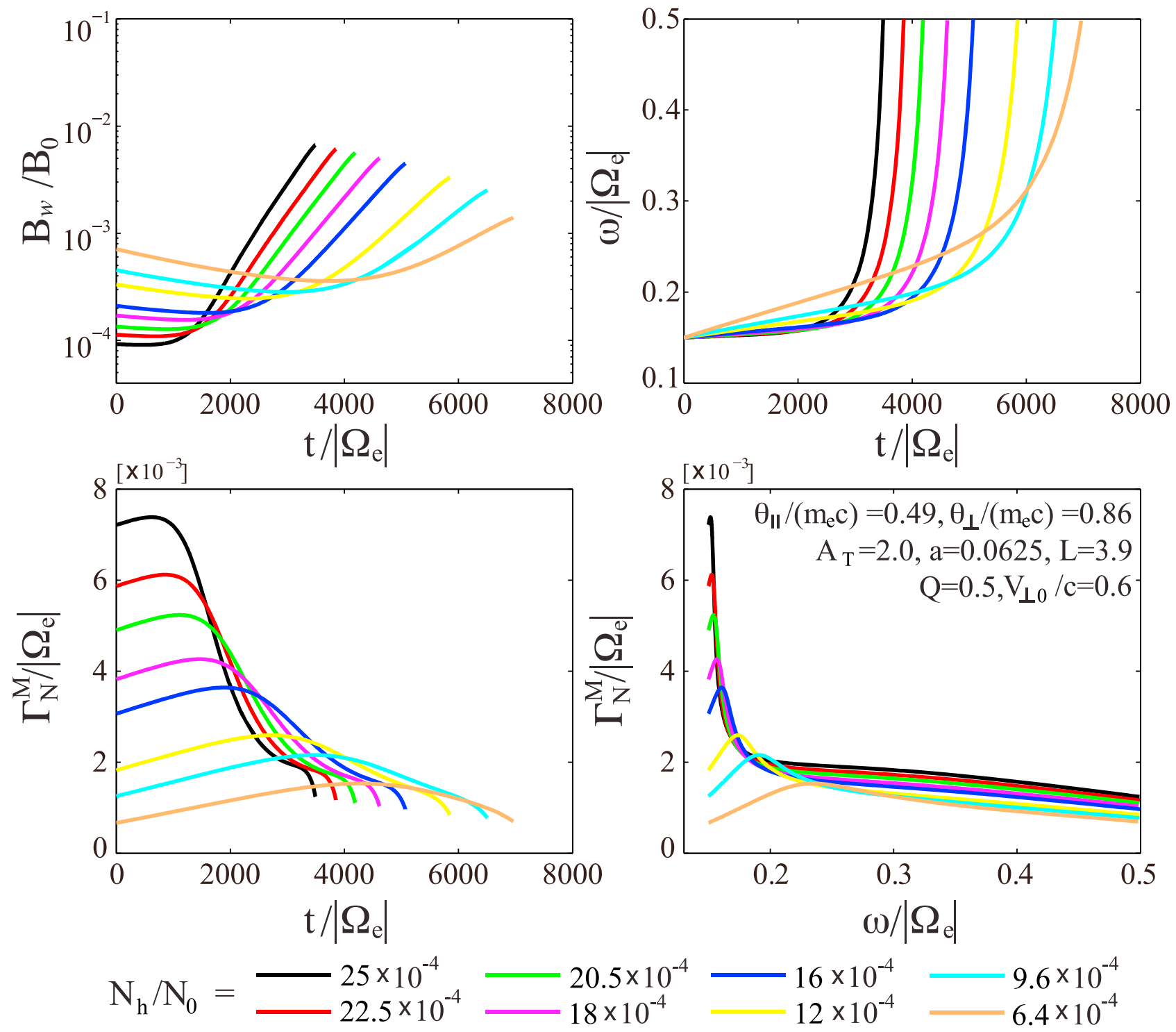

Figure 11. Solutions to the whistler mode wave equations (45) and (46) at the equator, corresponding to the particle distribution (63) for the indicated values of the hot electron number density $N_{h}$.

imum value of the corresponding relativistic linear growth rate.

[36] We obtain the maximum linear convective wave gain $g_{L}^{M}$ corresponding to the bi-Maxwellian distribution (60) as

$\frac{g_{L}^{M}}{\lambda_{L}^{M}}=\frac{1.05 \times 10^{5}}{L^{2}}\left(\frac{N_{h}}{N_{0}}\right) \frac{1}{a}\left(\frac{m_{e} c}{\theta_{\|}}\right)\left\{\frac{y_{m} I_{4}\left(x_{m}, y_{m}\right)-x_{m} I_{3}\left(x_{m}, y_{m}\right)}{y_{m}^{2}}\right\}$,

where $x_{m}, y_{m}$ are evaluated at the frequency at which the linear growth rate (61) maximizes. The maximum nonlinear convective wave gain $g_{N}^{M}$ corresponding to distribution (63) is given by

$$
\begin{aligned}
\frac{g_{N}^{M}}{\lambda_{N}^{M}}= & \frac{7.5 \times 10^{3}}{L^{2}}\left(\frac{N_{h}}{N_{0}}\right) \frac{1}{a}\left(\frac{m_{e} c}{\theta_{\|}}\right)\left(\frac{\xi}{\tilde{B}_{w} \tilde{\omega}}\right)^{1 / 2} \frac{Q \chi^{3 / 2}}{\tilde{\gamma}_{R}^{3 / 2}}\left(\frac{V_{\perp 0}}{c}\right)^{3 / 2} \\
& \cdot \exp \left(-\frac{\tilde{p}_{R}^{2}}{\theta_{\|}^{2}}\right)
\end{aligned}
$$

where the relevant quantities on the right-hand side of (67) are evaluated at the frequency at which the nonlinear growth rate $\Gamma_{N}^{M}$ maximizes.

[37] In Figure 12 we plot profiles of $g_{L}^{M} / \lambda_{L}^{M}$ and $g_{N}^{M} / \lambda_{N}^{M}$ as functions of $N_{h} / N_{0}$. In Figure 12 (middle) we use the parameters adopted in Figures 10 and 11, for which $A_{T}=$ 2.0. In Figures 12 (top) and 12 (bottom) we use the same parameters except that we set $\theta_{\perp} /\left(m_{e} c\right)=0.57, A_{T}=0.32$ and $\theta_{\perp} /\left(m_{e} c\right)=1.11, A_{T}=3.99$, respectively. In each panel, for the appropriate parameter values, we set $B_{w}(0) / B_{0}$ 

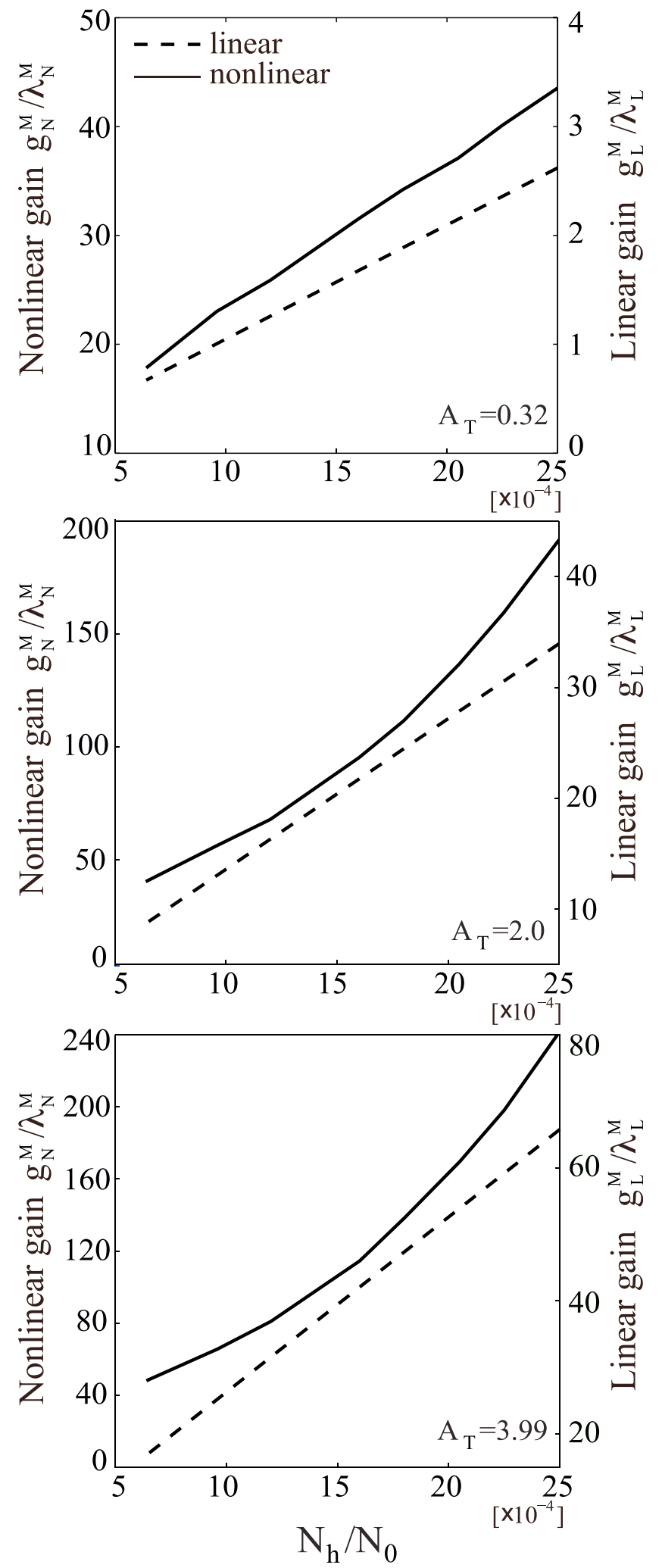

Figure 12. Linear wave gain $g_{L}^{M} / \lambda_{L}^{M}$ given by (66) and nonlinear wave gain $g_{N}^{M} / \lambda_{N}^{M}$ given by (67), each plotted as functions of the hot electron number density $N_{h}$. (middle) Parameter values are those adopted in Figures 10 and 11 . (top) Parameter values as in Figure 12 (middle), except $\theta_{\perp} /$ $\left(m_{e} c\right)=0.57, A_{T}=0.32$. (bottom) Parameter values as in Figure 12 (middle), except $\theta_{\perp} /\left(m_{e} c\right)=1.11, A_{T}=3.99$. to be slightly greater than the numerical threshold value, and $\omega(0)$ to equal the frequency at which the linear growth rate (61) maximizes. The panels indicate that for a given value of $N_{h} / N_{0}$ both $g_{N}^{M} / \lambda_{N}^{M}$ and $g_{L}^{M} / \lambda_{L}^{M}$ increase as the thermal anisotropy $A_{T}$ is increased. In all panels we find $g_{N}^{M} / \lambda_{N}^{M} \gg g_{L}^{M} / \lambda_{L}^{M}$ which implies that the corresponding extreme electron flux is much less than that predicted from Kennel-Petschek theory.

\section{Summary}

[38] The Kennel-Petschek concept of self-limitation of radiation belt particle fluxes was reexamined by Summers et al. [2009] in a fully relativistic regime. We have extended this study and have obtained new relativistic limiting forms for the omnidirectional electron integral flux (19) and electron differential flux ((17) and (27)). These forms are simple, explicit, and involve the particle pitch angle anisotropy $A_{\text {rel }}$, the wave gain $G$, and the convective growth length $H$. The limiting forms further depend on the spectral index $l$ and pitch angle index $s$ of the assumed particle distribution (1), as well as on the cold plasma parameter $a=\left|\Omega_{e}\right|^{2} / \omega_{p e}^{2}$. We compared our limiting solution for the electron differential flux with measured fluxes at geosynchronous orbit. For some parameter values, the experimental fluxes were reasonably close to the limiting solution, though a strict comparison is difficult since empirical values for $s$ were unavailable. In common with previous studies of the Kennel-Petschek limit on stably trapped particle fluxes, our analysis (in section 2) is based on linear wave growth theory.

[39] We have incorporated (in sections 3-6) the effects of nonlinear wave growth in the determination of extreme radiation belt particle fluxes. We assume that electromagnetic whistler mode waves generated in the vicinity of the equator undergo a linear growth phase, in which the wave frequency is constant, followed by a nonlinear growth phase in which the wave frequency is time-increasing. To calculate nonlinear wave growth rates we used the nonlinear theory of Omura et al. [2009] for field-aligned whistler mode waves. We solved the coupled "chorus equations" ((45) and (46)) at the equator to obtain the wave amplitude $B_{w}$ and wave frequency $\omega$, and we hence obtained the nonlinear wave growth rate $\Gamma_{N}$. We expressed the total power gain $G_{T O T}$ (given by (52)) as the sum of the linear wave gain $g_{L}$, during the linear growth phase, and the nonlinear wave gain $g_{N}$ during the nonlinear growth phase. We determined $G_{T O T}$ using the maximum values of the linear and nonlinear growth rates, and thereby obtained $G_{T O T}$ as a function of the energetic electron number density $N_{h}$. $G_{T O T}$ depends on the convective length scales $\lambda_{L} H, \lambda_{N} H$ for linear, nonlinear wave growth. We derived results (58) and (59) for the extreme values of the electron integral flux and differential flux, in which $N_{h}$ takes on an extreme value corresponding to a specified value of the maximum total wave gain $G_{T O T}$; the extreme value of $N_{h}$ must be obtained numerically. The theoretical results (58) and (59) for the extreme flux in the nonlinear regime can be regarded as generalizations of the classical Kennel-Petschek limits which are based on linear wave growth. We calculated the wave gains $g_{L} / \lambda_{L}, g_{N} / \lambda_{N}$ corresponding to the distributions (29) and (40), and also the wave gains $g_{L}^{M} / \lambda_{L}^{M}, g_{N}^{M} / \lambda_{N}^{M}$ corresponding to the distributions (60) and (63). In cases of 
moderate thermal anisotropy in which the maximum nonlinear wave growth rate is much greater than the maximum linear growth rate, our study implies that the inclusion of nonlinear wave effects sharply reduces the prediction of extreme radiation belt electron flux in comparison to that predicted by Kennel-Petschek theory. Inclusion of nonlinear wave effects can likewise be expected to modify considerably the treatment of other radiation belt phenomena traditionally analyzed by linear or quasi-linear theories. In this regard an interesting future project would be to examine how the inclusion of nonlinear wave growth effects impacts the well-known result of Schulz and Davidson [1988] that the limiting radiation belt differential flux varies asymptotically as $1 / E$ at large energy $E$ in the nonrelativistic regime. Our present study does not include explicitly the nonlinear effects that lead to the saturation of nonlinear wave growth at the magnetic equator. A further challenging project would be to analyze the nonlinear wave-particle interaction effects that lead to this saturation process.

\section{Appendix A: Integrals Occurring in Equations (11) and (12)}

[40] The integrals $I_{1}$ and $I_{2}$ occurring in equations (11) and (12) are given by

$$
\begin{gathered}
I_{1}(x, y)=\int_{0}^{\infty} \frac{d z z^{2 s+1}\left[2(l+1) z^{2}-2 s \bar{p}_{R}^{2}\right]}{\Delta_{R}\left(z^{2}+\bar{p}_{R}^{2}\right)^{l+s+2}}, \\
I_{2}(x, y)=\int_{0}^{\infty} \frac{d z}{\Delta_{R} \gamma_{R}} \frac{2 s\left(-\bar{p}_{R}\right) z^{2 s+1}}{\left(z^{2}+\bar{p}_{R}^{2}\right)^{l+s+1}},
\end{gathered}
$$

where

$$
\begin{gathered}
\gamma_{R}=\frac{-x+y\left[\left(y^{2}-x^{2}\right)\left(1+z^{2}\right)+1\right]^{1 / 2}}{y^{2}-x^{2}}, \\
\bar{p}_{R}=\frac{\gamma_{R} x-1}{y}, \\
\Delta_{R}=1-\frac{x\left(\gamma_{R} x-1\right)}{\gamma_{R} y^{2}},
\end{gathered}
$$

where $x$ and $y$ are defined by (8), and satisfy equation (7).

\section{Appendix B: Nonrelativistic Forms of Results (17), (19), and (20)}

[41] In the nonrelativistic regime, we set $\gamma=1, p=m_{e} v$, $E=\left(m_{e} v^{2}\right) / 2, p_{*}=m_{e} v_{*}, E_{*}=\left(m_{e} v_{*}^{2}\right) / 2$. The nonrelativistic forms corresponding to results (17), (19), and (20) are

$$
\begin{aligned}
J_{\perp}\left(p_{*}\right)= & \frac{B_{0}}{2 \pi^{3} e} \frac{1}{\left(m_{e} c\right)}\left(\frac{m_{e} c}{p_{*}}\right)^{2 l} \frac{\Gamma(l+s+1)}{\Gamma(s+1) \Gamma(l)} \frac{\left(1-x_{m}\right)^{2 l-1}}{y_{m}^{2(l-1)}} \\
& \cdot \frac{G}{\left(\tilde{A}-A_{C}\right) H},
\end{aligned}
$$

$$
\begin{gathered}
I_{4 \pi}\left(E>E_{*}\right)=\Lambda \frac{c B_{0}}{\pi^{3 / 2} e} \frac{G}{\left(\tilde{A}-A_{C}\right) H}, \\
\Lambda=\frac{1}{2(l-1)}\left(\frac{m_{e} c^{2}}{2 E_{*}}\right)^{l-1} \frac{\Gamma(l+s+1)}{\Gamma\left(s+\frac{3}{2}\right) \Gamma(l)} \frac{\left(1-x_{m}\right)^{2 l-1}}{y_{m}^{2(l-1)}},
\end{gathered}
$$

where

$$
\begin{gathered}
\tilde{A}=s, \\
\frac{p_{*}}{m_{e} c}=\frac{-a^{1 / 2}}{\left[s(1+s)^{2}+a s^{2}\right]^{1 / 2}},
\end{gathered}
$$

and

$$
\frac{E_{*}}{m_{e} c^{2}}=\frac{a}{2\left[s(1+s)^{2}+a s^{2}\right]} .
$$

The nonrelativistic form of the limiting perpendicular differential electron flux is given by equations (27), (B1), (B4) and (B5).

\section{Appendix C: Integrals Occurring in Equations (61) and (66)}

[42] The integrals occurring in equations (61) and (66) are given by

$$
I_{3}(x, y)=\int_{0}^{\infty} \frac{z^{3} e^{-z^{2}}}{\Delta_{R}^{M}} \exp \left[-\left(\frac{1-\gamma_{R}^{M} x}{y}\right)^{2}\left(\frac{m_{e} c}{\theta_{\|}}\right)^{2}\right] d z,
$$

$$
\begin{aligned}
I_{4}(x, y)= & \left(\frac{\theta_{\perp}^{2}}{\theta_{\|}^{2}}-1\right) \int_{0}^{\infty} \frac{z^{3} e^{-z^{2}}}{\Delta_{R}^{M} \gamma_{R}^{M}}\left(\frac{1-\gamma_{R}^{M} x}{y}\right) \\
& \cdot \exp \left[-\left(\frac{1-\gamma_{R}^{M} x}{y}\right)^{2}\left(\frac{m_{e} c}{\theta_{\|}}\right)^{2}\right] d z,
\end{aligned}
$$

where

$$
\begin{gathered}
\gamma_{R}^{M}=\frac{-x+y\left[\left(y^{2}-x^{2}\right)\left\{1+z^{2}\left(\frac{\theta_{\perp}}{m_{e} c}\right)^{2}\right\}+1\right]^{1 / 2}}{y^{2}-x^{2}}, \\
\Delta_{R}^{M}=1-\frac{x\left(\gamma_{R}^{M} x-1\right)}{\gamma_{R}^{M} y^{2}},
\end{gathered}
$$

where $x$ and $y$ are defined by (8), and satisfy equation (7).

[43] Acknowledgments. We thank T. Paul O'Brien for providing experimental data on the electron differential flux at geosynchronous orbit used in Figure 5. This work is supported by a Discovery Grant of the Natural Sciences and Engineering Research Council of Canada to D.S. and by the WCU grant R31-10016 funded by the Korean Ministry of Education, Science, and Technology. Additional support is acknowledged from grantin-aid 20340135 of the Ministry of Education, Science, Sports and Culture of Japan. 
[44] Robert Lysak thanks the reviewers for their assistance in evaluating this paper.

\section{References}

Baker, D. N. (2002), How to cope with space weather, Science, 297(5586), 1486-1487.

Baker, D. N., and S. G. Kanekal (2008), Solar cycle changes, geomagnetic variations, and energetic particle properties in the inner magnetosphere, J. Atmos. Sol. Terr. Phys., 70, 195-206.

Baker, D. N., et al. (1997), Recurrent geomagnetic storms and relativistic electron enhancements in the outer magnetosphere: ISTP coordinated measurements, J. Geophys. Res., 102(A7), 14,141-14,148.

Baker, D. N., T. I. Pulkkinen, X. Li, S. G. Kanekal, J. B. Blake, R. S. Selesnick, M. G. Henderson, G. D. Reeves, H. E. Spence, and G. Rostoker (1998), Coronal mass ejections, magnetic clouds, and relativistic magnetospheric electron events: ISTP, J. Geophys. Res., 103(A8), 17,279-17,291.

Chum, J., O. Santolik, A. W. Breneman, C. A. Kletzing, D. A. Gurnett, and J. S. Pickett (2007), Chorus source properties that produce time shifts and frequency range differences observed on different Cluster spacecraft, J. Geophys. Res., 112, A06206, doi:10.1029/2006JA012061.

Fennell, J. F., H. C. Koons, M. W. Chen, and J. B. Blake (2000), Internal charging: A preliminary environmental specification for satellites, IEEE Trans. Plasma Sci., 28(6), 2029-2036.

Friedel, R. H. W., G. D. Reeves, and T. Obara (2002), Relativistic electron dynamics in the inner magnetosphere-A review, J. Atmos. Sol. Terr. Phys., 64, 265-282.

Hikishima, M., S. Yagitani, Y. Omura, and I. Nagano (2009), Full particle simulation of whistler-mode rising chorus emissions in the magnetosphere, J. Geophys. Res., 114, A01203, doi:10.1029/2008JA013625.

Horne, R. B. (2002), The contribution of wave-particle interactions to electron loss and acceleration in the Earth's radiation belts during geomagnetic storms, in Review of Radio Science, 1999-2002, edited by W. R. Stone, chap. 33, pp. 801-828, IEEE Press, Piscataway, N. J.

Hudson, M. K., B. T. Kress, H.-R. Mueller, J. A. Zastrow, and J. B. Blake (2008), Relationship of the Van Allen radiation belts to solar wind drivers, J. Atmos. Sol. Terr. Phys., 70, 708-729.

Kataoka, R., and Y. Miyoshi (2008), Average profiles of the solar wind and outer radiation belt during the extreme flux enhancement of relativistic electrons at geosynchronous orbit, Ann. Geophys., 26, 1335-1339.

Katoh, Y., and Y. Omura (2011), Amplitude dependence of frequency sweep rates of whistler mode chorus emissions, J. Geophys. Res., 116, A07201, doi:10.1029/2011JA016496.

Kennel, C. F., and H. E. Petschek (1966), Limit on stably trapped particle fluxes, J. Geophys. Res., 71(1), 1-28.

Li, W., R. M. Thorne, V. Angelopoulos, J. W. Bonnell, J. P. McFadden, C. W. Carlson, O. LeContel, A. Roux, K. H. Glassmeier, and H. U. Auste (2009), Evaluation of whistler-mode chorus intensification on the nightside during an injection event observed on the THEMIS spacecraft, J. Geophys. Res., 114, A00C14, doi:10.1029/2008JA013554.

Mauk, B. H., and N. J. Fox (2010), Electron radiation belts of the solar system, J. Geophys. Res., 115, A12220, doi:10.1029/2010JA015660.

Millan, R. M., and R. M. Thorne (2007), Review of radiation belt relativistic electron losses, J. Atmos. Sol. Terr. Phys., 69, 362-377.

Nagano, I., X.-Y. Wu, S. Yagitani, K. Miyamura, and H. Matsumoto (1998), Unusual whistler with very large dispersion near the magnetopause: Geotail observation and ray-tracing modeling, J. Geophys. Res., 103(A6), 11,827-11,840

O’Brien, T. P., K. R. Lorentzen, I. R. Mann, N. P. Meredith, J. B. Blake, J. F. Fennell, M. D. Looper, D. K. Milling, and R. R. Anderson (2003), Energization of relativistic electrons in the presence of ULF power and MeV microbursts: Evidence for dual ULF and VLF acceleration, J. Geophys. Res., 108(A8), 1329, doi:10.1029/2002JA009784.
O’Brien, T. P., J. F. Fennell, J. L. Roeder, and G. D. Reeves (2007) Extreme electron fluxes in the outer zone, Space Weather, 5, S01001, doi:10.1029/2006SW000240.

Omura, Y., and D. Summers (2006), Dynamics of high-energy electrons interacting with whistler mode chorus emissions in the magnetosphere, J. Geophys. Res., 111, A09222, doi:10.1029/2006JA011600.

Omura, Y., Y. Katoh, and D. Summers (2008), Theory and simulation of the generation of whistler-mode chorus, J. Geophys. Res., 113, A04223, doi:10.1029/2007JA012622.

Omura, Y., M. Hikishima, Y. Katoh, D. Summers, and S. Yagitani (2009), Nonlinear mechanisms of lower-band and upper-band VLF chorus emissions in the magnetosphere, J. Geophys. Res., 114, A07217, doi:10.1029/ 2009JA014206.

Omura, Y., and D. Nunn (2011), Triggering process of whistler-mode chorus emissions in the magnetosphere, J. Geophys. Res., 116, A05205, doi:10.1029/2010JA016280.

Reeves, G. D., D. N. Baker, R. D. Belian, J. B. Blake, T. E. Cayton, J. F. Fennell, R. H. W. Friedel, M. M. Meier, R. S. Selesnick, and H. E. Spence (1998), The global response of relativistic radiation belt electrons to the January 1997 magnetic cloud, Geophys. Res. Lett., 25, 3265-3268.

Schulz, M., and G. T. Davidson (1988), Limiting energy spectrum of a saturated radiation belt, J. Geophys. Res., 93(A1), 59-76.

Sheeley, B. W., M. B. Moldwin, H. K. Rassoul, and R. R. Anderson (2001), An empirical plasmasphere and trough density model: CRRES observations, J. Geophys. Res., 106(A11), 25,631-25,641.

Shprits, Y. Y., S. R. Elkington, N. P. Meredith, and D. A. Subbotin (2008), Review of modeling of losses and sources of relativistic electrons in the outer radiation belt I: Radial transport, J. Atmos. Sol. Terr. Phys., 70, 1679-1693.

Summers, D., and C. Ma (2000), A model for generating relativistic electrons in the Earth's inner magnetosphere based on gyroresonant waveparticle interactions, J. Geophys. Res., 105(A2), 2625-2639.

Summers, D., R. M. Thorne, and F. Xiao (1998), Relativistic theory of wave-particle resonant diffusion with application to electron acceleration in the magnetosphere, J. Geophys. Res., 103(A9), 20,487-20,500.

Summers, D., B. Ni, and N. P. Meredith (2007a), Timescales for radiation belt electron acceleration and loss due to resonant wave-particle interactions: 1. Theory, J. Geophys. Res., 112, A04206, doi:10.1029/2006JA011801.

Summers, D., B. Ni, and N. P. Meredith (2007b), Timescales for radiation belt electron acceleration and loss due to resonant wave-particle interactions: 2. Evaluation for VLF chorus, ELF hiss, and electromagnetic ion cyclotron waves, J. Geophys. Res., 112, A04207, doi:10.1029/2006JA011993.

Summers, D., R. Tang, and R. M. Thorne (2009), Limit on stably trapped particle fluxes in planetary magnetospheres, J. Geophys. Res., 114, A10210, doi:10.1029/2009JA014428.

Thorne, R. M., R. B. Horne, S. A. Glauert, N. P. Meredith, Y. Shprits, D. Summers, and R. R. Anderson (2005a), The influence of wave-particle interactions on relativistic electron dynamics during storms, in Inner Magnetosphere Interactions: New Perspectives From Imaging, Geophys. Monogr. Ser., vol. 159, edited by J. Burch, M. Schulz, and H. Spence, pp. 101-112, AGU, Washington, D. C.

Thorne, R. M., T. P. O'Brien, Y. Y. Shprits, D. Summers, and R. B. Horne (2005b), Timescale for $\mathrm{MeV}$ electron microburst loss during geomagnetic storms, J. Geophys. Res., 110, A09202, doi:10.1029/2004JA010882.

Xiao, F., R. M. Thorne, and D. Summers (1998), Instability of electromagnetic R-mode waves in a relativistic plasma, Phys. Plasmas, 5, 2489-2497.

Y. Omura, Research Institute for Sustainable Humanosphere, Kyoto University, Uji, Kyoto 611-0011, Japan. (omura@rish.kyoto-u.ac.jp)

D. Summers and R. Tang, Department of Mathematics and Statistics, Memorial University of Newfoundland, St. John's, NL A1C 5S7, Canada. (dsummers@mun.ca; tangrx@gmail.com) 